\title{
Type-I cascaded quadratic soliton compression in lithium niobate: Compressing femtosecond pulses from high-power fiber lasers
}

Bache, Morten; Wise, Frank W.

Published in:

Physical Review A

Link to article, DOI:

10.1103/PhysRevA.81.053815

Publication date:

2010

Document Version

Publisher's PDF, also known as Version of record

Link back to DTU Orbit

Citation (APA):

Bache, M., \& Wise, F. W. (2010). Type-I cascaded quadratic soliton compression in lithium niobate: Compressing femtosecond pulses from high-power fiber lasers. Physical Review A, 81(5), 053815. https://doi.org/10.1103/PhysRevA.81.053815

\section{General rights}

Copyright and moral rights for the publications made accessible in the public portal are retained by the authors and/or other copyright owners and it is a condition of accessing publications that users recognise and abide by the legal requirements associated with these rights.

- Users may download and print one copy of any publication from the public portal for the purpose of private study or research.

- You may not further distribute the material or use it for any profit-making activity or commercial gain

- You may freely distribute the URL identifying the publication in the public portal 


\title{
Type-I cascaded quadratic soliton compression in lithium niobate: Compressing femtosecond pulses from high-power fiber lasers
}

\author{
Morten Bache ${ }^{1, *}$ and Frank W. Wise ${ }^{2}$ \\ ${ }^{1}$ DTU Fotonik, Department of Photonics Engineering, Technical University of Denmark, DK-2800 Kgs. Lyngby, Denmark \\ ${ }^{2}$ Department of Applied and Engineering Physics, Cornell University, Ithaca, New York 14853, USA
}

(Received 15 December 2009; published 11 May 2010)

\begin{abstract}
The output pulses of a commercial high-power femtosecond fiber laser or amplifier are typically around 300-500 fs with wavelengths of approximately $1030 \mathrm{~nm}$ and tens of microjoules of pulse energy. Here, we present a numerical study of cascaded quadratic soliton compression of such pulses in $\mathrm{LiNbO}_{3}$ using second-harmonic generation in a type-I phase-matching configuration. We find that because of competing cubic material nonlinearities, compression can only occur in the nonstationary regime, where group-velocity-mismatch-induced Raman-like nonlocal effects prevent compression to less than $100 \mathrm{fs}$. However, the strong group-velocity dispersion implies that the pulses can achieve moderate compression to durations of less than $130 \mathrm{fs}$ in available crystal lengths. Most of the pulse energy is conserved because the compression is moderate. The effects of diffraction and spatial walk-off are addressed, and in particular the latter could become an issue when compressing such long crystals (around $10 \mathrm{~cm}$ long). We finally show that the second harmonic contains a short pulse locked to the pump and a long multi-picosecond red-shifted detrimental component. The latter is caused by the nonlocal effects in the nonstationary regime, but because it is strongly red-shifted to a position that can be predicted, we show that it can be removed using a bandpass filter, leaving a visible component of less than $100 \mathrm{fs}$ at $\lambda=515 \mathrm{~nm}$ with excellent pulse quality.
\end{abstract}

DOI: 10.1103/PhysRevA.81.053815 PACS number(s): 42.65.Re, 05.45.Yv, 42.65.Ky, 42.70.Mp

\section{INTRODUCTION}

Pulsed fiber laser systems are currently undergoing rapid development, and by employing the chirped pulse amplification (CPA) technique, high-energy femtosecond pulses can be generated with microjoule to millijoule energies [1]. Combined with the fact that the fiber laser technology offers a rugged, cheap, and compact platform, ultrafast fiber CPA (fCPA) systems could compete with solid-state amplifier systems. However, the gain bandwidth of the Yb-doped fibers typically used for lasing in the $1.0-\mu \mathrm{m}$ region is considerably less than competing solid-state materials (such as Ti:sapphire crystals). Thus, due to the buildup of an excessive nonlinear phase shift, Yb-based fCPA lasers are often limited to a pulse duration that typically is sub-picosecond at best (around $500-700 \mathrm{fs}$ ) for $\sim 100-\mu \mathrm{J}$ pulses [2], while shorter pulses can be reached ( $\sim 250$ fs) for $\sim 30-\mu \mathrm{J}$ pulses [3].

Efficient external compression methods are therefore needed. A prototypical compressor consists of a piece of nonlinear material, where a broadening of the pulse bandwidth occurs by self-phase modulation (SPM), followed by a dispersive element (gratings or chirped mirrors) that provides temporal compression. With this method (using a short piece of fiber as nonlinear material) 27-fs sub- $\mu \mathrm{J}$ pulses were generated from $270-\mathrm{fs} 0.8-\mu \mathrm{J}$ pulses from an fCPA system [4]. Alternative methods consist of using long ( $0.5 \mathrm{~m}$ or more) gas cells or filaments [5] as nonlinear material, and this works with pulse energies from $50 \mu \mathrm{J}$ to around $1 \mathrm{~mJ}$ (limited in part by self-focusing effects) or possibly even greater energies [6].

When soliton compression is used, both the SPM-induced pulse broadening and dispersion-induced compression occur in the same material [7]. The SPM is provided by the Kerr self-

\footnotetext{
${ }^{*}$ moba@ fotonik.dtu.dk
}

focusing nonlinearity inherent in most materials. However, as self-focusing solitons require anomalous dispersion, this can only be achieved in the near-infrared (IR) region through strong waveguide dispersion. This means using specially designed fibers, such as microstructured fibers. Fibers have a very limited maximum pulse energy of a few nanojoules, albeit large mode-area microstructured solid-core and hollow-core fiber compressors can support up to $1 \mu \mathrm{J}$ [8].

Unfortunately, the pulse energy from fCPA systems lies exactly in the gap between these methods. Here, we study a compression method that can compensate for this. It is a soliton compressor based on cascaded quadratic nonlinearities [9-11]; see Fig. 1. This has several advantages: As it relies on a self-defocusing nonlinearity, there are no problems with selffocusing effects, and pulse energies of multiple millijoules can be compressed. Moreover, solitons of the self-defocusing kind require normal instead of anomalous dispersion, implying that solitons can be generated in the visible and near-IR regions. Finally, it is extremely simple, as it relies on just a small piece of quadratic nonlinear crystal, preceded only by a lens or a beam expander [12].

The basis for the cascaded quadratic soliton compressor (CQSC) is phase-mismatched second-harmonic generation (SHG). The cascaded energy transfer from the pump (fundamental wave, $\mathrm{FW}$ ) to the second harmonic $(\mathrm{SH})$ and back imposes a strong SPM-like nonlinear phase shift on the FW, whose sign can be made self-defocusing [13,14]. Thereby, the FW pulse can be compressed with normal dispersion [9], and soliton compression becomes possible in the visible and near-IR regions [10].

In this article, we investigate the CQSC in a lithium niobate $\left(\mathrm{LiNbO}_{3}, \mathrm{LN}\right)$ crystal cut for type-I phase matching, where the goal is to perform moderate compression of longer femtosecond pulses from fCPA systems at the $\mathrm{Yb}$ gain wavelength of $1030 \mathrm{~nm}$. We show that in order to overcome the detrimental 


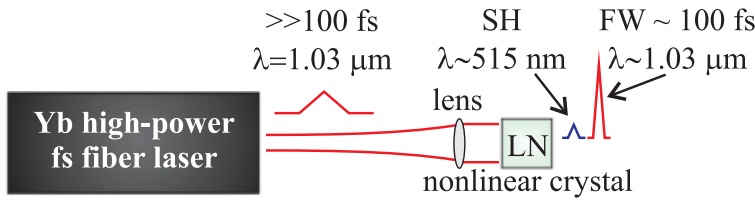

FIG. 1. (Color online) Cascaded quadratic soliton compressor studied here: The $\mathrm{Yb}$ fiber laser produces energetic longer pulses $(\gg 100 \mathrm{fs})$ that are launched collimated in a quadratic nonlinear NB crystal, where the phase-mismatched type-I SHG process compresses the input pulse.

cubic nonlinearities, the phase mismatch has to be chosen low enough that the compression occurs in the so-called nonstationary regime. This regime is dominated by group-velocitymismatch (GVM) effects, and large GVM is a well-known drawback of using LN in the near-IR region for SHG. However, when only moderate compression is desired, the soliton order can be kept low, and we show through numerical simulations that reasonable pulse quality can be achieved and that up to $80 \%$ of the pulse energy is retained in the central spike. The compression limit is found to be around $120 \mathrm{fs}$ full width at half maximum (FWHM), which is a limit set by the GVM effects. The compression occurs in a crystal of reasonable length, $10 \mathrm{~cm}$. This is possible only because LN has a very large second-order dispersion. Finally, we show that bandpass filtering of the SH actually can lead to a very clean visible pulse of less than $100 \mathrm{fs}$ with around $0.1 \%$ conversion efficiency.

In this article, we first discuss the general compression properties of LN in a cascaded type-I SHG interaction setup in Sec. II and then in Sec. III show some numerical simulations of pulses coming from two different commercially available fCPA systems. We conclude in Sec. IV. The properties of LN are discussed in Appendix A, and in Appendix B we discuss the anisotropic Kerr nonlinear response of LN. In Appendixes $\mathrm{C}$ and $\mathrm{D}$, we discuss the conversion relations between Gaussian and SI units for cubic nonlinear coefficients and Miller's rule, respectively.

\section{TYPE-I COMPRESSION PROPERTIES OF LITHIUM NIOBATE CRYSTALS}

With the CQSC, high-energy, few-cycle compressed pulses can be generated, as was experimentally observed at $1250 \mathrm{~nm}$ [15]. However, the first studies performed at $800 \mathrm{~nm}$ were plagued by GVM effects that prevented reaching the few-cycle regime $[9,10,15]$. These studies used a $\beta$-bariumborate $(\mathrm{BBO})$ crystal in a type-I SHG $o o \rightarrow e$ configuration, where the FW (ordinary polarization) is orthogonal to the SH (extraordinary polarization) and where birefringent phase matching is possible by angle-tuning the crystal. BBO is in many respects an ideal nonlinear crystal: it has low dispersion, a very large transparency window, and a reasonably strong quadratic nonlinearity relative to the detrimental cubic one. As we have shown in previous theoretical and numerical studies, $\mathrm{BBO}$ provides an excellent compression of longer pulses to ultrashort duration at the $\mathrm{Yb}$ gain wavelengths [16-18]. The problems with BBO are that good-quality waveguides are not supported and that it is very difficult to grow long crystals. In particular, the latter is important if only moderate compression of longer pulses is desired. In moderate soliton compression, most of the pulse energy is conserved in the compressed pulse, and the pulse has a reduced pedestal. The problem is that compression will only occur after a long propagation length.

We therefore turn here to LN, which is a widely used quadratic nonlinear crystal for IR frequency conversion. $\mathrm{LN}$ is attractive due to extremely large quadratic nonlinearities that can be accessed through a quasi-phase-matched (QPM) type-0 SHG phase-matching configuration where $\mathrm{FW}$ and $\mathrm{SH}$ have identical polarizations. However, here we study LN in a type-I configuration as BBO. The advantages of a type-I configuration over QPM are very flexible phase-matching properties and a simpler device, and in type-I LN the effective quadratic nonlinearity is still more than twice as large as in BBO.

LN is usually not considered very suitable for SHG of short pulses in the near-IR because the SH becomes very dispersive; thus, the $\mathrm{FW}$ and $\mathrm{SH}$ group velocities are very different, resulting in large GVM. This is also why LN has not been used in the near-IR as nonlinear medium for the CQSC, for which GVM is a very detrimental effect. Another disadvantage for the CQSC is that the Kerr nonlinear response is several times larger than $\mathrm{BBO}$, which counteracts the advantage of the large quadratic nonlinearity of LN. Therefore, the CQSC experiments done so far using LN were done in the telecommunication band and exploited QPM in a type- 0 configuration [19], where effective quadratic nonlinearity is around three times larger than what can be achieved in a type-I configuration. However, we now show that type-I LN offers a quite decent compression performance without having to custom design a QPM grating.

\section{A. Solitons with cascaded quadratic nonlinearities}

In cascaded SHG, we study the frequency conversion $\omega_{1}+\omega_{1} \rightarrow \omega_{2}$ in the absence of phase matching. Here, $\omega_{j}$ are the frequencies of the $\mathrm{FW}(j=1)$ and $\mathrm{SH}(j=2)$, and by energy conversion $\omega_{2}=2 \omega_{1}$. During this interaction, the FW effectively experiences a Kerr-like nonlinear refractive index. This is in addition to the cubic (Kerr) nonlinearities that are always present in all media. We can write the total refractive index of the FW [see Eq. (C2)] as

$$
n=n_{1}+\frac{1}{2}\left|\mathcal{E}_{1}\right|^{2} n_{\text {cubic }}=n_{1}+I_{1} n_{\text {cubic }}^{I},
$$

where $n_{1}$ is the FW linear refractive index, $\mathcal{E}_{1}$ is the FW electric field, and $I_{1}$ is the FW intensity. It is typical to report the nonlinear refractive index relative to the electric field, $n_{\text {cubic }}$, or to the intensity, $n_{\text {cubic }}^{I}$. For simplicity, we have here neglected cross-phase modulation (XPM) contributions since they are small in cascaded SHG. As mentioned, we have contributions from both cascaded quadratic and cubic Kerr nonlinearities,

$$
n_{\text {cubic }}^{I}=n_{\mathrm{SHG}}^{I}+n_{\mathrm{Kerr}, 11}^{I},
$$

where $n_{\text {Kerr,11 }}^{I}$ is the SPM Kerr nonlinear refractive index of the FW (see Appendix B for details on the notation). The contribution from the cascaded quadratic nonlinearities can in the large phase-mismatch limit $(\Delta k L \gg 1$, where $L$ is the crystal length) be approximated as [13]

$$
n_{\mathrm{SHG}}^{I} \simeq-\frac{2 \omega_{1} d_{\mathrm{eff}}^{2}}{c^{2} \varepsilon_{0} \lambda_{1} n_{1}^{2} n_{2} \Delta k},
$$


where $d_{\text {eff }}$ is the effective $\chi^{(2)}$ nonlinearity. For $\Delta k=k_{2}-$ $2 k_{1}>0$, the cascaded contribution is negative, that is, selfdefocusing. Here, $k_{j}=n_{j} \omega_{j} / c$ is the wave number.

The effective quadratic nonlinearity of the type-I $o o \rightarrow e$ interaction for the negative uniaxial $3 m$ crystal class (LN, $\mathrm{BBO})$ is

$$
d_{\mathrm{eff}}=d_{31} \sin \theta-d_{22} \cos \theta \sin 3 \phi,
$$

where the angles are defined in Fig. 10 in Appendix B. Choosing $\phi=-\pi / 2$ gives maximum nonlinearity (see Appendix A).

In cascaded quadratic soliton compression, the aim is to get $n_{\mathrm{SHG}}^{I}<0$ and $\left|n_{\mathrm{SHG}}^{I}\right|>n_{\mathrm{Kerr}, 11}^{I}$ so as to achieve a total self-defocusing cubic nonlinearity. The soliton interaction can then be described by an effective soliton order [17],

$$
N_{\mathrm{eff}}^{2}=N_{\mathrm{SHG}}^{2}-N_{\mathrm{Kerr}}^{2}=\frac{\omega_{1}}{c} L_{D, 1} I_{\mathrm{in}}\left(\left|n_{\mathrm{SHG}}^{I}\right|-n_{\mathrm{Kerr}, 11}^{I}\right),
$$

where $N_{\mathrm{SHG}}=L_{D, 1} I_{\text {in }}\left|n_{\mathrm{SHG}}^{I}\right| \omega_{1} / c$ is the soliton order of the self-defocusing cascaded quadratic nonlinearity, and $N_{\text {Kerr }}=$ $L_{D, 1} I_{\mathrm{in}} n_{\mathrm{Kerr}, 11}^{I} \omega_{1} / c$ is the soliton order of the material Kerr self-focusing cubic nonlinearity. The FW dispersion length is $L_{\mathrm{D}, 1}=T_{\mathrm{in}}^{2} /\left|k_{1}^{(2)}\right|$, where $k_{1}^{(2)}$ is the FW group-velocity dispersion (GVD) and $T_{\text {in }}$ is the input pulse duration. We use the following notation for the dispersion: $k_{j}^{(m)}=d^{m} k_{j} /\left.d \omega^{m}\right|_{\omega=\omega_{j}}$.

\section{B. Linear and nonlinear response of $\mathrm{LN}$ at $1.03 \mu \mathrm{m}$}

For $\lambda_{1}=1.03 \mu \mathrm{m}$, the operating wavelength of most Yb-based fiber laser amplifiers, the properties of $\mathrm{LN}$ are
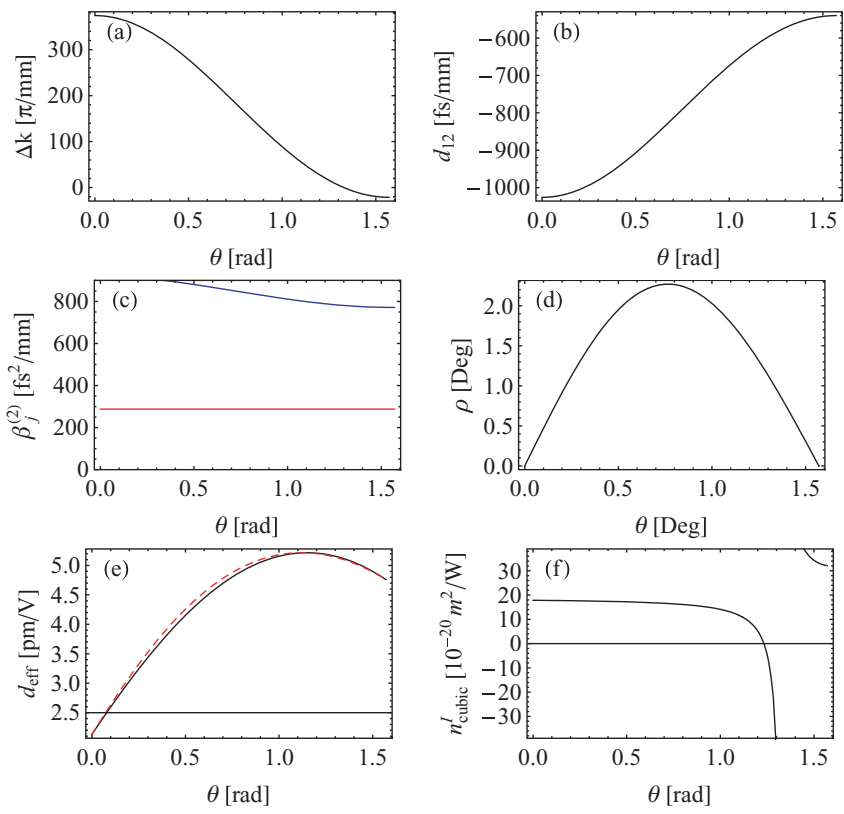

FIG. 2. (Color online) Properties at $\lambda_{1}=1.03 \mu \mathrm{m}$ when angletuning the LN crystal: (a) phase mismatch, (b) GVM parameter, (c) GVD of FW (red) and SH (blue), and (d) the spatial walk-off angle $\rho$. The effective quadratic nonlinearities neglecting (black) and including (dashed red) spatial walk-off are shown in (e) and (f) are the total cubic Kerr nonlinearity, Eq. (2), from cascaded quadratic nonlinearities and Kerr SPM (using $n_{\text {Kerr }}^{I}=18 \times 10^{-20} \mathrm{~m}^{2} / \mathrm{W}$; see Appendix B). summarized in Fig. 2: The phase mismatch (a) becomes small at $\theta \simeq 1.3 \mathrm{rad}$ (70-75 deg). As shown in (e), in this range $d_{\text {eff }} \simeq 5.2 \mathrm{pm} / \mathrm{V}$, and the total nonlinear refractive index (f), as expressed by Eq. (2), can become negative, implying that the cascaded nonlinearity is stronger than the Kerr nonlinearity. This happens for $\Delta k<62 \mathrm{~mm}^{-1}$ (or $\theta>70.4 \mathrm{deg}$ ). At $\theta=$ $75.8 \mathrm{deg}$, phase matching is achieved, after which $n_{\mathrm{SHG}}^{I}>0$ and thus is self-focusing.

GVM is very large [see Fig. 2(b)], which as we see later sets a strong limitation on the compression performance. The GVD is shown in Fig. 2(c), and importantly FW GVD (red) is large and normal (i.e., positive). It stays normal until $\lambda_{1}>1.9 \mu \mathrm{m}$, after which it becomes anomalous and self-defocusing solitons are no longer supported. The SH GVD (blue) is about three times larger than the FW GVD.

Since the type-I critical phase matching is employed, the walk-off angle $\rho=\arctan \left[\tan (\theta) n_{o}^{2} / n_{e}^{2}\right]-\theta$ (valid for a negative uniaxial crystal) is nonzero; see Fig. 2(d). In Fig. 2(e), it is apparent that $d_{\text {eff }}$ is largely unaffected by walk-off. However, walk-off does set a limit on the effective interaction length between the pump and the $\mathrm{SH}$, as we discuss later.

\section{Compression diagram for type-I LN}

We now generalize to other wavelengths and summarize the type-I compression performance of LN in Fig. 3. ${ }^{1}$ This

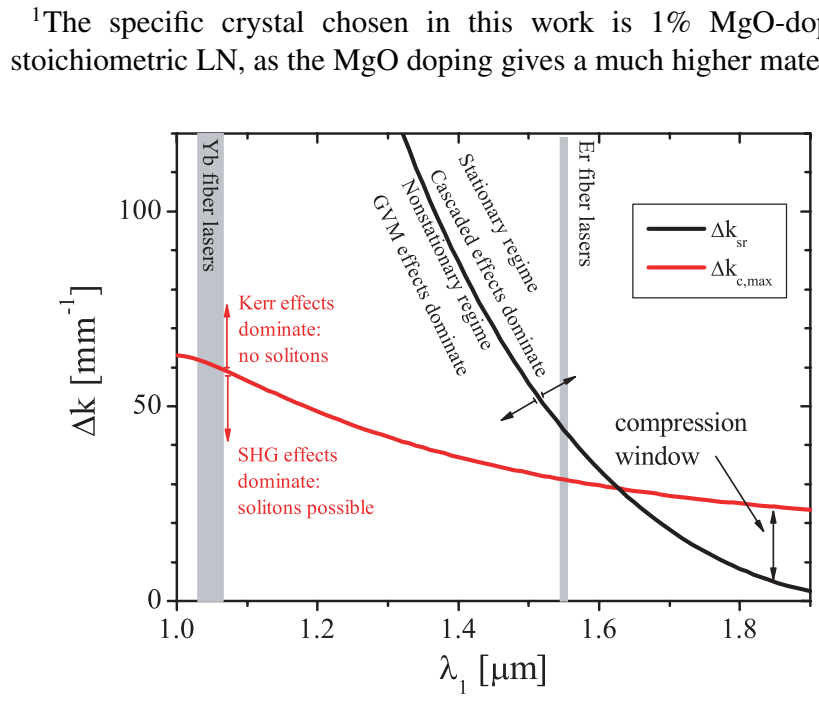

FIG. 3. (Color online) Compression diagram for $1 \% \mathrm{MgO}: \mathrm{sLN}$ at room temperature and aligned for type-I SHG. For various pump wavelengths $\lambda_{1}$, the choice of phase-mismatch parameter $\Delta k$ affects the compression. In order to excite solitons, the phase mismatch must be kept below the red line $\left(\Delta k<\Delta k_{c, \max }\right)$, because otherwise the material cubic nonlinearities are too strong $\left(n_{\mathrm{Kerr}, 11}^{I}>\left|n_{\mathrm{SHG}}^{I}\right|\right)$. Optimal compression occurs when the cascaded nonlinearities dominate over GVM effects ( $\Delta k>\Delta k_{\mathrm{sr}}$, above the black line). We have also indicated the operation wavelengths of $\mathrm{Yb}$ - and Er-doped fiber lasers. The red line uses Miller's rule to estimate the nonlinear quadratic and cubic susceptibilities at other wavelengths, cf. Eqs. (D1) and (D2), and uses $n_{\text {Kerr, } 11}^{I}=20 \times 10^{-20} \mathrm{~m}^{2} / \mathrm{W}$ for $\lambda=0.78 \mu \mathrm{m}$ (see Appendix B for an extended discussion). 
compression diagram shows the different compression regimes for the CQSC as the wavelength and the phase mismatch is varied. Above the red curve, the total nonlinear refractive index is focusing $n_{\text {cubic }}^{I}>0$, so solitons are not supported since the FW GVD is normal. The curve is found by setting $\left|n_{\mathrm{SHG}}^{I}\right|=$ $n_{\text {Kerr,11 [17]: }}^{I}$

$$
\Delta k_{c, \max }=k_{1} \frac{2 d_{\mathrm{eff}}^{2}}{c \varepsilon_{0} n_{1}^{2} n_{2} n_{\mathrm{Kerr}, 11}^{I}} .
$$

Below the black curve, the compression performance is dominated by GVM effects (nonstationary regime), while above it is dominated by cascaded effects (stationary regime). The curve is to the second order ${ }^{2}$ given by [16],

$$
\Delta k_{\mathrm{sr}}=\frac{d_{12}^{2}}{2 k_{2}^{(2)}},
$$

where $d_{12}=k_{1}^{(1)}-k_{2}^{(1)}$ is the GVM parameter and $k_{2}^{(2)}$ is the SH GVD. It is better when the curve is lower because this implies that the chance of observing solitons in the stationary regime increases. Thus, the very large GVM parameter $d_{12}$ is detrimental because it pushes the curve upward. Instead, the huge SH GVD values [see Fig. 2(c)] are actually helping to push the curve downward. Therefore, a large SH GVD can actually be beneficial for clean soliton compression.

The optimal compression occurs in the so-called compression window [16], where the soliton compressor works most efficiently because solitons are supported in the stationary regime. The diagram shows a compression window for type-I LN in the regime $\lambda_{1}=1.6-1.9 \mu \mathrm{m}$. Unfortunately, in this range there are no fCPA systems. Fortunately, as we show, in the nonstationary regime compression is possible as long as the effective soliton order is low enough.

Coming back to $\lambda_{1}=1.03 \mu \mathrm{m}$, we observe that solitons are supported for when $0 \ll \Delta k<\Delta k_{c \text {, max }}=62 \mathrm{~mm}^{-1}$. When getting too close to $\Delta k_{c, \max }$, the intensities required to observe solitons become very large, implying excessive Kerr XPM effects and increased Raman-like GVM effects [18]. On the other hand, for $\Delta k$ that is too small, the cascading limit ceases to hold, and also the compressor performance decreases due to excessive GVM effects [18]. In fact, generally the compression limit in the nonstationary regime (in which the system will always be for $\Delta k \sim 0$ ) is roughly given by the pulse duration for which $L_{\mathrm{coh}}=L_{\mathrm{GVM}}$, where $L_{\mathrm{coh}}=\pi /|\Delta k|$ is the coherence length and $L_{\mathrm{GVM}}=\Delta t_{\text {soliton }} /\left|d_{12}\right|$ is the dynamic GVM length of a sech-shaped soliton. By "dynamic," we mean that the GVM length changes as the soliton compresses. Thus, in the nonstationary regime the limit is ${ }^{3}$

$$
\Delta t_{\text {limit }}^{\mathrm{FWHM}} \sim 2 \ln (1+\sqrt{2}) \frac{\pi\left|d_{12}\right|}{|\Delta k|},
$$

damage threshold. Also, 5\% MgO-doped congruent LN would work well. See Appendix A for more details about the crystal.

${ }^{2} \mathrm{~A}$ more accurate transition easily can be calculated numerically using the full $\mathrm{SH}$ dispersion operator [18], which we have done in what follows.

${ }^{3}$ Note that this expression differs with a factor of $\pi / 2$ from the limit $T_{R, \mathrm{SHG}}=2\left|d_{12} / \Delta k\right|$ that we suggested in [18]; this is purely an empirical choice. where the factor in front of the fraction is the conversion factor to FWHM for a sech-shaped pulse. Obviously, as $\Delta k$ approaches the phase-matching point, the soliton cannot compress to short durations. We numerically found the optimal compression point in the $\Delta k=35-50 \mathrm{~mm}^{-1}$ regime, and the best results were for $\Delta k=45 \mathrm{~mm}^{-1}$, for which $n_{\mathrm{SHG}}^{I} \simeq$ $25 \times 10^{-20} \mathrm{~m}^{2} / \mathrm{W}$.

\section{Predicting the compression performance}

The next step is to estimate what the compression performance could look like. Here, the scaling laws ${ }^{4}$ come into the picture, which can be used to predict the propagation distance for optimal compression $z_{\text {opt }}$, the compression factor $f_{c}$, and the pulse quality $Q_{c}$ [17].

As we have pointed out recently [16], it is the phase mismatch and the GVM (zero- and first-order dispersions) that really control the compression properties. The only requirement to the second-order dispersion is that FW GVD is normal $k_{1}^{(2)}>0$ so as to support solitons. Otherwise, as we discuss later, the FW GVD is basically just determining the optimum compression length. The SH GVD instead plays a minor role in the compression properties; cf. Eq. (7). Our initial idea was to exploit the fact that $\mathrm{LN}$ is quite dispersive when pumped at $\lambda_{1} \sim 1.0 \mu \mathrm{m}$, so the very large FW GVD makes it possible to compress the pulse in a short crystal.

So why and when is it interesting to increase GVD as to compress in a short crystal? Obviously, the crystals have length limits, which for LN is around $100 \mathrm{~mm}$. The optimal compression point scales as [17]

$$
\frac{z_{\mathrm{opt}}}{z_{0}}=\frac{0.44}{N_{\mathrm{eff}}}+\frac{2.56}{N_{\mathrm{eff}}^{3}}-0.002,
$$

where $z_{0}=\frac{\pi}{2} L_{\mathrm{D}, 1}$ is the soliton length [20]. Thus, the point where the pulse compression is optimal depends on the effective soliton order, the input pulse duration, and the FW GVD. Therefore, since quality LN crystals are at most $100 \mathrm{~mm}$ long, the CQSC works best when the soliton order is large and the GVD length is short. When the soliton order is large, the detrimental effects due to GVM are strongly increased [15,18], in particular in the nonstationary regime. Therefore, in the case we study here, clean compression can only be done with low soliton order, and therefore the FW GVD must be large to ensure compression in realistic crystal lengths.

A downside to the large GVD is the following: Given that some effective soliton order is required, then since $N_{\text {eff }} \propto \sqrt{I_{\text {in }} L_{\mathrm{D}, 1}} \propto T_{\text {in }} \sqrt{I_{\text {in }} /\left|k_{1}^{(2)}\right|}$, a large GVD gives a short GVD length and thus larger intensities are needed to excite a soliton. The same problem is found for short-input pulses, say, from a Ti:sapphire amplifier. However, this is only an issue if operating with intensities close to the damage threshold, which is not the case here: The intensities are moderate $\left(I_{\text {in }} \ll 100 \mathrm{GW} / \mathrm{cm}^{2}\right)$, and instead our issue is to get the solitons to compress in a crystal that is not too long.

\footnotetext{
${ }^{4}$ Note that the scaling laws presented here are only approximate figures when used in the nonstationary regime because they were found in the stationary regime.
} 
The compression factor $f_{c}=T_{\text {in }} / \Delta t_{\mathrm{opt}}$, where $\Delta t_{\mathrm{opt}}$ is the pulse compressed pulse duration at $z_{\mathrm{opt}}$, is also affected by the effective soliton order [17]:

$$
f_{c}=4.7\left(N_{\text {eff }}-0.86\right) \text {. }
$$

The pulse quality can also be predicted and is defined as the ratio between the compressed pulse fluence with that of the input pulse. It scales as [17]

$$
Q_{c}=\left[0.24\left(N_{\mathrm{eff}}-1\right)^{1.11}+1\right]^{-1} .
$$

We can use this to calculate the compressed pulse peak intensity $I_{\mathrm{opt}}=Q_{c} f_{c} I_{\mathrm{in}}$ and energy $E_{\mathrm{opt}}=Q_{c} E_{\mathrm{in}}$. An advantage of using low soliton orders is that $Q_{c}$ remains high, and thus the compressed pulse retains most of the initial pulse energy.

\section{E. Compression performance of fCPA systems}

Let us use these scaling laws to predict the compression performance of fCPA systems. High-energy femtosecond pulses from fCPA systems use both Yb-doped and Er-doped gain fibers. Since fCPA systems are diode pumped with a wavelength just less than $1.0 \mu \mathrm{m}$, the quantum efficiency of $\mathrm{Yb}$-doped systems is greater, and therefore the majority of commercial and scientific systems prefer to use $\mathrm{Yb}$ over Er. Most systems operate at the $\lambda=1.03 \mu \mathrm{m} \mathrm{Yb}$ emission line and can for low pulse energies $(<15 \mu \mathrm{J})$ generate pulses as short as $250 \mathrm{fs}$, whereas greater pulse energies result in longer pulses (currently 50- $\mu \mathrm{J} 450$-fs pulses are the state-of-the-art for commercial systems). In Er amplifier systems, much lower pulse energies are available, typically pulses of $1-3 \mu \mathrm{J}$ and 500-700 fs at $\lambda=1.55 \mu \mathrm{m}$; such low pulse energies and long pulse duration mean that only very low soliton orders can be excited, and thus the CQSC can only achieve very moderate compression in very long crystals.

The basis for the following case studies and numerical simulations is therefore a couple of commercially available Yb-based fCPA systems, both operating at $1030 \mathrm{~nm}$. Case 1 is a Clark MXR Impulse ${ }^{5}$ giving $15-\mu \mathrm{J} 250$-fs FWHM pulses, which represents a system giving quite short yet still reasonably energetic pulses as a starting point. Case 2 is an Amplitude Systemes Tangerine ${ }^{6}$ giving 50- $\mu$ J 450-fs FWHM pulses, which represents a system with more energetic but also longer pulses.

The two cases are studied together, taking $\Delta k=45 \mathrm{~mm}^{-1}$. Figure 4(a) shows that in case 1 we need to focus the pulses to $w_{0}<600 \mu \mathrm{m}$ to observe solitons: In this regime, Fig. 4(d) shows that the Rayleigh length $z_{R}=\pi w_{0}^{2} / \lambda$ is only five to six times larger than the optimal compression point $z_{\mathrm{opt}}$ of around $100 \mathrm{~mm}$. This is borderline for experiencing diffraction problems. Even increasing or decreasing the waist does not improve this ratio much. In case 2 instead, the increased pulse energy makes solitons appear already at $w_{0} \simeq 1.6 \mathrm{~mm}$, despite the longer pulse duration. This means that diffraction should be less of an issue: In Fig. 4(d), the pulse compression

\footnotetext{
${ }^{5}$ http://www.clark-mxr.com

${ }^{6} \mathrm{http}: / /$ www.amplitude-systemes.com
}
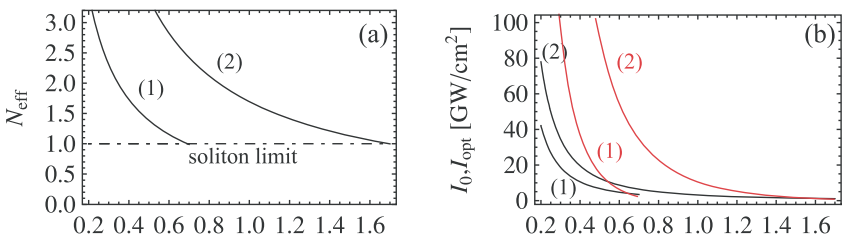

$w_{0}[\mathrm{~mm}]$
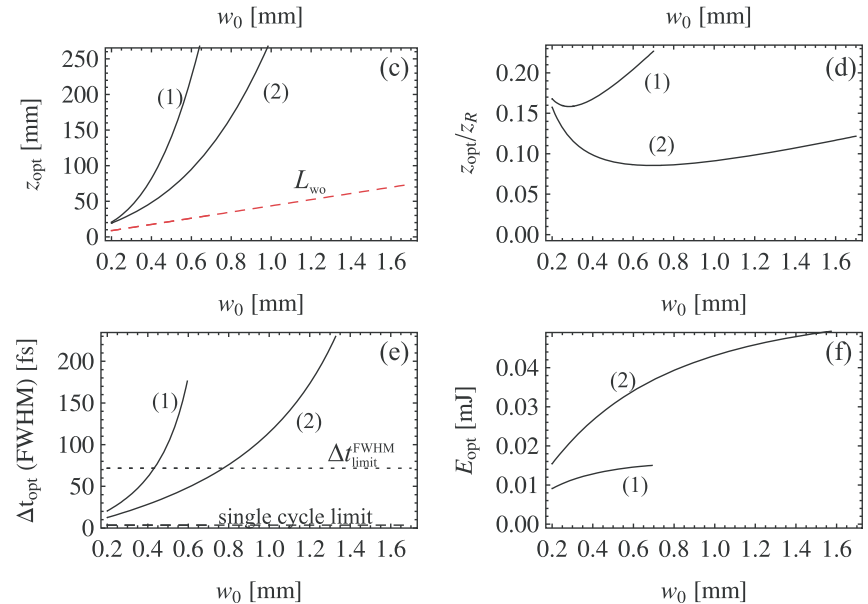

FIG. 4. (Color online) Practical operation range of the LN type-I compression system at $\lambda_{1}=1.03 \mu \mathrm{m}$ for $\Delta k=45 \mathrm{~mm}^{-1}$. The plots show the predicted behavior when the FW waist $w_{0}$ is varied. The two cases are (1) pump pulses with $T_{\mathrm{in}}^{\mathrm{FWHM}}=250 \mathrm{fs}$ and $15 \mu \mathrm{J}$ pulse energy and (2) pump pulses with $T_{\text {in }}^{\mathrm{FWHM}}=450 \mathrm{fs}$ and $50 \mu \mathrm{J}$ pulse energy. The curves in (b)-(f) are calculated based on $N_{\text {eff }}$ shown in (a) by using the scaling laws [17] that hold for $N_{\text {eff }}>1$.

point relative to the Rayleigh length of the focused beam is significantly smaller in case 2 .

Figure 4(c) indicates that the spatial walk-off in the crystal can become an issue: The crystal should be shorter than the spatial walk-off length $L_{\mathrm{wo}}=w_{0} / \tan \rho \simeq w_{0} / \rho$ to ensure proper interaction between the FW and the SH, but evidently the pulse compression lengths in both cases are longer by factors of at least two or three than the spatial walk-off length. Therefore, it might be necessary to compensate for this by using two crystals, one inverted relative to the other, so that the walk-off direction in the second crystal is inverted with respect to the first crystal [21].

An alternative solution to the walk-off problem is to turn to a noncritical phase-matching scheme, where $\rho=0$. This happens for $\theta=0$ or $\pi / 2$; see Fig. 2(d). Of course, this removes the possibility of tuning the phase matching via $\theta$, and one has to turn to temperature tuning of $\Delta k$. The temperature needed to get to the desired operation point ( $\Delta k \simeq 40-50 \mathrm{~mm}^{-1}$ ) can be estimated using the temperaturedependent Sellmeier equations [22], and our calculations indicate that it should happen already at a temperature of around $45^{\circ} \mathrm{C}$. This would make an easy solution to the walk-off problem.

The strong GVM implies that compression of Yb-based systems can only occur in the nonstationary regime; see Fig. 3. Thus, unless $N_{\text {eff }}$ is close to unity, the GVM-induced Ramanlike effects dominate, and the FW pulse becomes extremely distorted and very poorly compressed. Actually, it almost never makes sense to use $N_{\text {eff }}$ larger than what is sufficient to reach the limit expressed by Eq. (8), and typically even an $N_{\text {eff }}$ 
smaller than that. The limit is drawn as a dotted line in Fig. 4(e), and it is reached around $w_{0}=400 \mu \mathrm{m}$ in case 1 and $w_{0}=$ $800 \mu \mathrm{m}$ in case 2 .

Finally, Fig. 4(b) shows that quite moderate input intensities must be used to achieve solitons in both cases. This is related to the quite long input pulse durations. Furthermore, Fig. 4(f) shows that the low soliton orders conserve most of the pulse energy in both cases.

\section{NUMERICAL SIMULATIONS}

We here present numerical simulations of the two cases using a plane-wave temporal model based on the slowly evolving wave equation (see more details in [17] and references therein), which includes self-steepening effects and higher order dispersion. This model is justified as long as diffraction is minimal, which we assume is the case when the crystal length is much shorter than the Rayleigh length and when spatial walk-off is minimal. This requirement is discussed further later.

\section{A. Case 1: 250-fs 15- $\mu \mathrm{J}$ pulses}

For the 250 -fs $15-\mu \mathrm{J}$ pulses from a Clark laser system, we found that the best compression was obtained with $N_{\text {eff }} \sim$ 1.3-1.5. This soliton order can be achieved with $15-\mu \mathrm{J}$ pulse energy when the pump is focused to around $w_{0}=400 \mu \mathrm{m}$; see Fig. 4(a).

The theoretical compression factor for such soliton orders is $f_{c}=2-3$, that is, a $\Delta t_{\text {opt }} \sim 80-125$ fs FWHM compressed pulse is predicted. In Fig. 5, we show the results of a simulation with $N_{\text {eff }}=1.4$. This soliton order gave the best compression: A slightly asymmetric $\Delta t_{\text {opt }}=126 \mathrm{fs}$ (FWHM) pulse is observed after $91 \mathrm{~mm}$ of propagation; see Fig. 5(a) and cut in (e). Preceeding the compression, note the SPM-like broadening of the FW spectrum in (b). It becomes slightly asymmetric with the blue shoulder being strongest, which is related to the Raman-like action of the cascaded nonlinearities $[18,23]$.

The observed compression is not quite as strong as predicted by the scaling law (10), but this is because the scaling laws are based on pulse compression in the stationary regime. On the other hand, the pulse quality is large, $Q_{c}=0.82$, so most of the pulse energy is retained in the central compressed part, and the pulse pedestal is also very small. These are the main advantages of soliton compression with low soliton orders.

In the SH time plot, Fig. 5(c), we observe the strong GVM first inducing a weak component and quickly escaping from the central part of the pulse, and later the GVM induces the characteristic dc-like trailing temporal pulse in the $\mathrm{SH}$ (this often occurs close to or at phase matching in the presence of GVM; see also [24]). This behavior is also reflected in the $\mathrm{SH}$ spectrum [see Fig. 5(d) and cut in (f)], which shows a very strong and extremely narrow red-shifted component building up, which eventually becomes the dominating contribution. As we discuss later, its spectral position can accurately be predicted by the nonlocal theory that was recently developed by us $[16,18]$. We believe that this strong and long SH trailing component actually causes the trailing part of the FW to be strongly depleted and that this is the main reason for the asymmetrical FW shape.

The question is now whether we can increase the effective soliton order and achieve further compression toward the limit predicted by Eq. (8). This turns out to be impossible: When $N_{\text {eff }}$ is increased, the GVM effects become stronger, making the compressed pulse more distorted. This is clearly observed in Fig. 6, where we increase $N_{\text {eff }}$ and compare it with the compression of Fig. 5: For $N_{\text {eff }}=2.0$, the compressed $\mathrm{FW}$ pulse in (a) is still quite short but clearly is less clean. For $N_{\text {eff }}=2.5$, the compressed pulse instead becomes quite distorted. It is also evident in the $\mathrm{SH}$ time plots that the trailing dc-like component increases with $N_{\text {eff }}$, while the central part
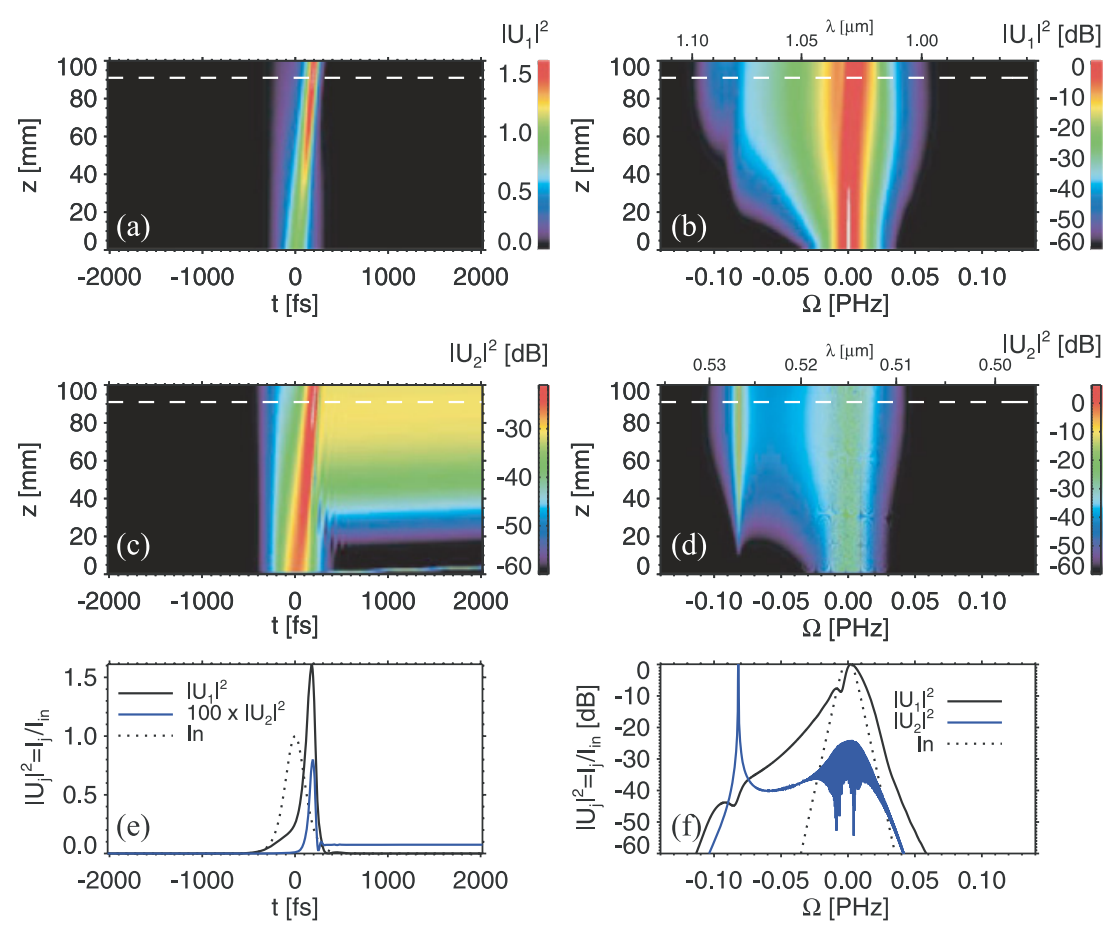

053815-6
FIG. 5. (Color online) Numerical simulation of soliton compression in $\mathrm{LN}$ with $\lambda_{1}=$ $1.03 \mu \mathrm{m}, T_{\text {in }}^{\mathrm{FWHM}}=250 \mathrm{fs}, \Delta k=45 \mathrm{~mm}^{-1}$, and $N_{\text {eff }}=1.4$ (implying $I_{\text {in }}=6.9 \mathrm{GW} / \mathrm{cm}^{2}$ ). The FW pulse shown in (a) compresses to $\Delta t_{\mathrm{opt}}=$ $126 \mathrm{fs}$ (FWHM) after propagating $91 \mathrm{~mm}$. The SH time plot (c) and FW (b) and SH (d) spectra are also shown on a logarithmic scale, and $U_{j}$ are normalized to the peak input FW electric field. In (e) and (f), cuts are shown at the optimal compression point $z=91 \mathrm{~mm}$ (corresponding to the white line in the two-dimensional plots). Note that the $\mathrm{SH}$ in (e) is magnified one hundred times. 

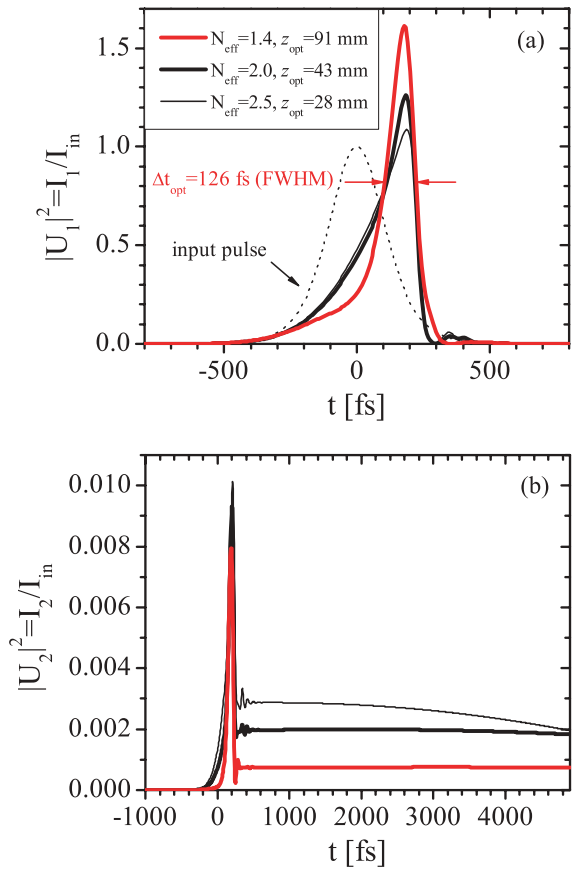

FIG. 6. (Color online) Simulations as in Fig. 5 but with increasing $N_{\text {eff }}$. The red curve corresponds to the optimal compression point from Fig. 5(e), whereas the black curves show what happens as the effective soliton order increases (making the optimal compression point occur sooner).

in all cases is a FWHM pulse less than 100 fs. It is quite weak because most of the converted SH energy is fed into the dc-like part of the pulse, which is connected to the strong spectral peak in the $\mathrm{SH}$ spectrum. This spectral peak becomes stronger with increased $N_{\text {eff }}$ (not shown) but does not change position as it does not depend on $N_{\text {eff }}$.

In order to understand the spectral content of the different temporal components, the cross-correlation frequencyresolved optical gating (XFROG) method is useful. The spectral strength is given by [25]

$$
S_{j}(z, T, \Omega)=\left|\int_{-\infty}^{\infty} \mathrm{d} t e^{i \Omega t} \mathcal{E}_{j}(z, t) \mathcal{E}_{\text {gate }}(t-T)\right|^{2},
$$

where $\mathcal{E}_{\text {gate }}(t)$ is a properly chosen gating pulse. The spectrograms of the compressed pulses in Fig. 6 for $N_{\text {eff }}=1.4$ are shown in Fig. 7. The FW compressed pulse is slightly blue-shifted (around $2 \mathrm{THz}$ ), and the compressed part (located at $T \sim 200 \mathrm{fs}$ ) shows a significantly broader spectrum.

The SH spectrum is very particular: The part of the pulse that propagates with the FW group velocity (the "locked" part) shows a quite clean short pulse. This group velocity locking of the SH has been observed before $[19,24]$ and can be understood from the nonlocal theory [16,18]: The SH has a component that is basically slaved to the FW due to the cascading nonlinearities. In frequency domain, it can be compactly expressed as [18]

$$
U_{2}(z, \Omega) \propto \tilde{R}_{-}(\Omega) \mathcal{F}\left[U_{1}^{2}(z, t)\right],
$$

where $\mathcal{F}\left[\right.$.] denotes the forward Fourier transform and $U_{j}$ are properly normalized fields. Thus, the spectral content of the $\mathrm{SH}$ is slaved to the spectral content of the spectrum of $U_{1}^{2}$.

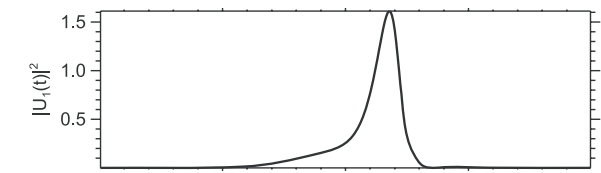

(a) $S_{1}$ (a.u.) $[d B]$
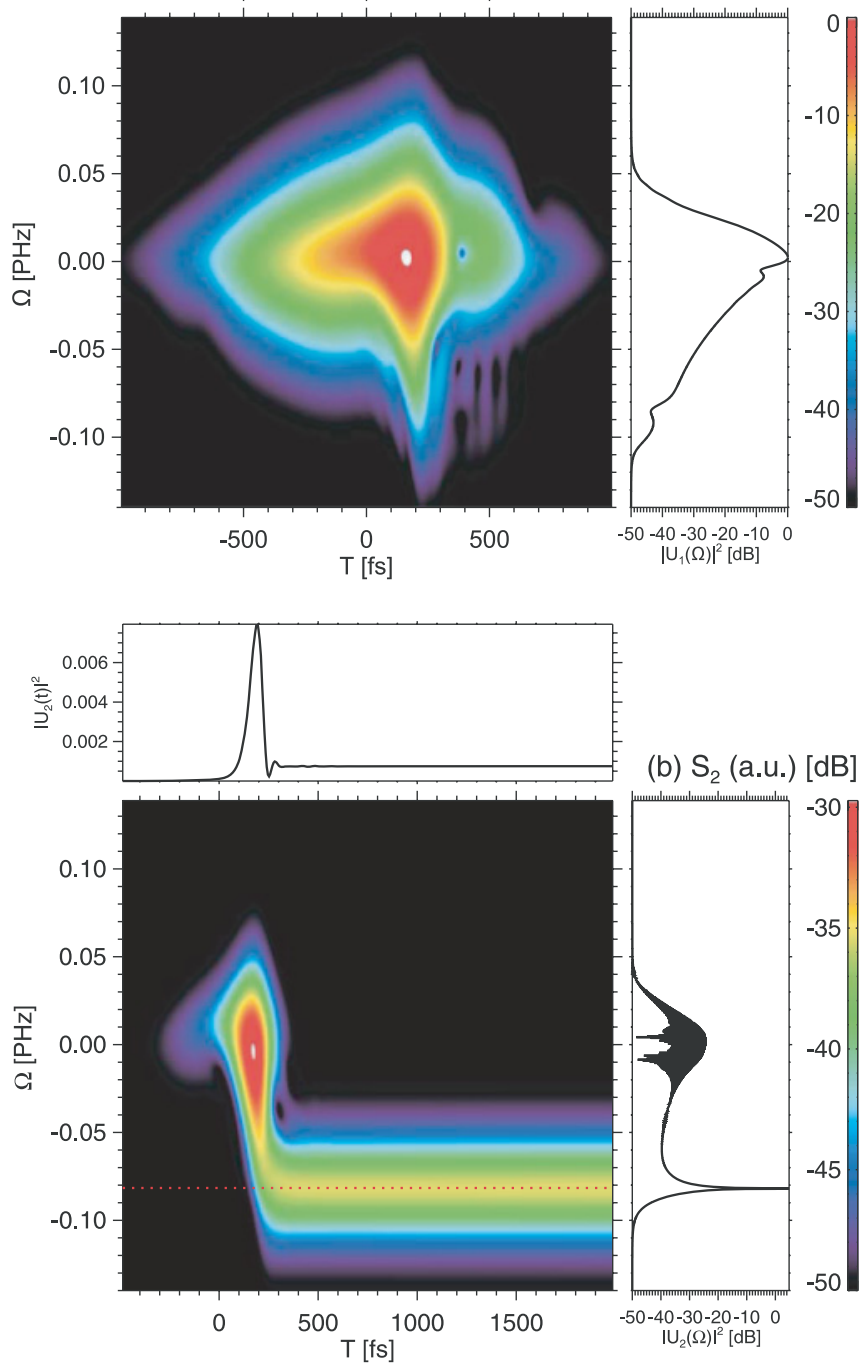

FIG. 7. (Color online) XFROG-like spectrograms of the simulation in Fig. 5 at the optimal compression point $z_{\text {opt }}=91 \mathrm{~mm}$. The sech-shaped gating pulse had $T_{0}^{\mathrm{FWHM}}=70 \mathrm{fs}$, and the spectrograms are normalized to the peak value of $S_{1}$. The top and side plots show the purely temporal and spectral traces, respectively, and are thus identical to Fig. 5(e) and 5(f). The red dashed line in (b) indicates the value $\Omega_{+}$as calculated by the nonlocal theory.

The weight is provided by the nonlocal Raman-like response function in the nonstationary regime [18],

$$
\tilde{R}_{-}(\Omega)=(2 \pi)^{-1 / 2} \frac{\Omega_{+} \Omega_{-}}{\left(\Omega-\Omega_{-}\right)\left(\Omega-\Omega_{+}\right)},
$$

where $\Omega_{ \pm}=\Omega_{a} \pm \Omega_{b}$. These frequencies can be calculated (to the second order) from the dispersion of the system as $\Omega_{a}=d_{12} / k_{2}^{(2)}=-1.044 \mathrm{PHz}$ and $\Omega_{b}=\mid 2 \Delta k / k_{2}^{(2)}-$ $\left.\Omega_{a}^{2}\right|^{1 / 2}=0.963 \mathrm{PHz}$. In the center around $\Omega=0$, where $\mathcal{F}\left[U_{1}^{2}(z, t)\right]$ is residing in this case, the response is quite flat. Thus, we get a SH component locked to the FW, and when the FW compresses, so does this SH component. 


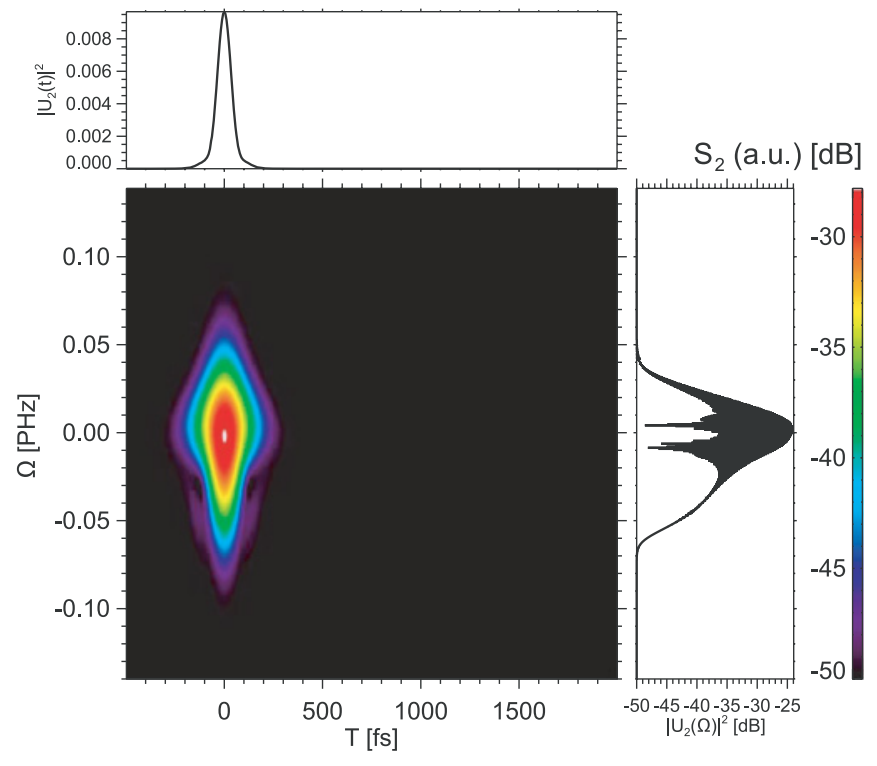

FIG. 8. (Color online) XFROG spectrogram of the SH pulse from Fig. 7(b) passed through a third-order super-Gaussian bandpass filter centered at the SH carrier frequency $\lambda_{2}=0.5150 \mu \mathrm{m}$ and with a FWHM of $100 \mathrm{THz}$.

Another striking feature of the SH spectrogram is the dc-like component: It is very evident as a long pulse is centered around $\Omega \sim-80 \mathrm{THz}$. Also, this peak can be understood from Eq. (13), because according to Eq. (14), the nonlocal response function in the nonstationary regime has sharp resonance peaks in the response at $\Omega=\Omega_{ \pm}$. By inserting the dispersion values of the simulation, we get $\Omega_{+}=-81.6 \mathrm{THz}$, in excellent correspondence with the observed peak position, as the red dashed line indicates. Instead, $\Omega_{-}$is located too far into the red side of the spectrum to affect the behavior.

Considering this spectral composition, filtering the disturbing SH component at $\Omega=\Omega_{+}$would leave a quite decent SH temporal pulse. In Fig. 8, we show that this is feasible: We pass the $\mathrm{SH}$ pulse through a super-Gaussian $(n=3)$ bandpass filter centered at $\omega_{2}$ and with a bandwidth of $100 \mathrm{THz}$ FWHM (corresponding to $15 \mathrm{~nm}$ ). This filters away the disturbing sharp peak, and a 80 -fs FWHM pulse remains at $\lambda=0.515 \mathrm{~nm}$. The peak intensity in this short pulse is around $0.006 I_{\text {in }}=0.0414 \mathrm{GW} / \mathrm{cm}^{2}$. If we assume that it is created with $15-\mu \mathrm{J}$ pulse energy focused to $w_{0}=0.5 \mathrm{~mm}$ to achieve $N_{\text {eff }}=1.4$, and that the generated $\mathrm{SH}$ has roughly the same spot size, then the pulse energy of the filtered 80 -fs pulse would be around $50 \mathrm{~nJ}$.

\section{B. Case 2: 450-fs 50- $\mu \mathrm{J}$ pulses}

In case 2 , the pulse duration is longer, $450 \mathrm{fs}$. When the pulse duration is longer, for a fixed soliton order the soliton will compress after a longer distance. This is because according to Eq. (9) $z_{\text {opt }} \propto L_{\mathrm{D}, 1} \propto T_{\mathrm{in}}^{2}$. However, we may compensate for this by increasing the effective soliton order enough to reach the limit governed by Eq. (8). For a $450-\mathrm{fs} 50-\mu \mathrm{J}$ pulse, it is achieved around $w_{0}=0.8 \mathrm{~mm}$ [see Fig. 4(e)], resulting in $N_{\text {eff }} \sim 2.0-2.5$. This higher soliton order should make it possible to compress in crystal lengths of around 10-15 cm; see Fig. 4(c).
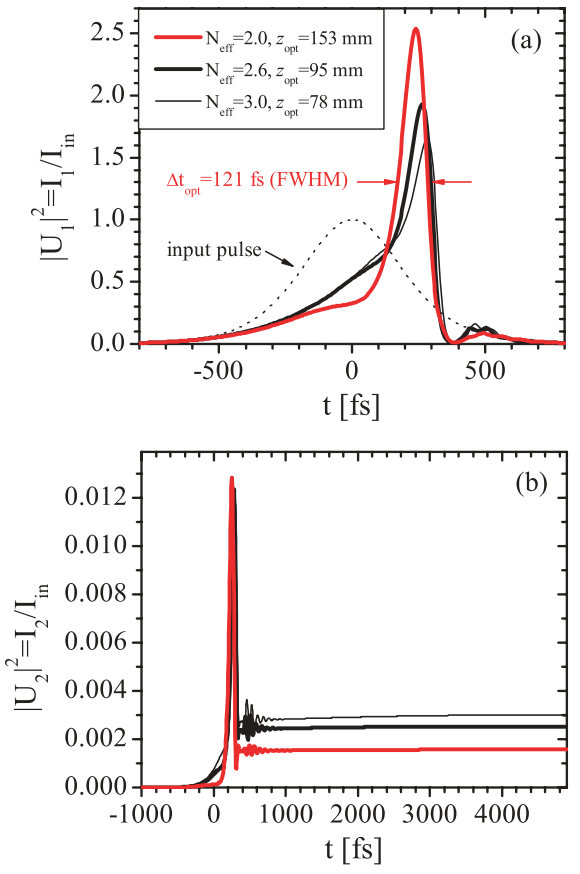

FIG. 9. (Color online) Numerical simulations using 450-fs 50- $\mu \mathrm{J}$ input pulses and taking $\Delta k=45 \mathrm{~mm}^{-1}$. The best pulse was observed for $N_{\text {eff }}=2.0$ (red curve) where pulse compression occurs after $15 \mathrm{~cm}$. The black curves show what happens as the effective soliton order increases (in which case the optimal compression point occurs sooner).

In Fig. 9, we show some numerical simulations using these longer, more energetic pulses. The best pulse observed shows a three-fold compression to $\Delta t_{\mathrm{opt}}=121 \mathrm{fs}$ (FWHM) at $N_{\text {eff }}=2.0$. The compression occurred after around $15 \mathrm{~cm}$ of propagation, so spatial walk-off would be an issue here. When the soliton order is increased to $N_{\text {eff }}=2.6$, the pulse becomes more distorted but still compresses to around 150 fs FWHM after $9.5 \mathrm{~cm}$, a more realistic interaction length. Finally, at $N_{\text {eff }}=3.0$, the pulse becomes too distorted as the GVM effects become stronger.

In the two cases, the pulses therefore eventually compress to the same duration, which is the limit imposed by the nonlocal GVM effects. The more energetic pulses in case 2 allow for a more defocused pump beam so the compression should be less affected by diffraction. On the other hand, as the pulses are longer, they compress later, so spatial walk-off is a more severe issue. A more optimal situation in both cases therefore would be more energetic pulses so that the pump can be defocused by a factor of two or three. This would diminish spatial walk-off effects.

\section{CONCLUSION}

Here we have shown that LN crystals in a type-I cascaded SHG interaction can provide moderate compression of femtosecond pulses from Yb-based fiber amplifier systems (1.03 $\mu \mathrm{m}$ wavelength). The phase mismatch was controlled through angle tuning (critical phase-matching interaction). By using numerical simulations, we found that the best compression was to around $120 \mathrm{fs} F W H$ after around $10 \mathrm{~cm}$ of propagation. 
Better compression was prevented in part by strong GVM effects, caused by strong dispersion in the LN crystal and competing material Kerr nonlinear effects. These are focusing in nature and counteract the defocusing Kerr-like nonlinearities from the cascaded SHG. In order to make the total nonlinear phase shift negative, the phase mismatch had to be taken quite low, and in this regime GVM effects dominate (the "nonstationary" regime). GVM imposes a strongly nonlocal temporal response in the cascaded nonlinearity that feeds most of the converted energy into a narrow red-shifted peak. In the temporal trace, this gave a SH with a multi-picosecond long trailing component. The FW therefore experienced a distorted compression unless the soliton order was kept very low. For such low soliton orders, the compression distance increases substantially, but here the strong dispersion of the LN crystal actually becomes an advantage: Due to a large GVD, the soliton dynamics occur in much shorter crystals than usual, and the numerics indicated compression in realistic crystal lengths $(10 \mathrm{~cm})$.

It was noted that using low soliton orders gave a compressed pulse retaining most of the input pulse energy (in the cases we showed around $80 \%$ ), and that the unavoidable soliton pedestal was less pronounced.

We also discussed the implications of using long crystals. Spatial walk-off will be an issue since a critical phasematching scheme is used that exploits birefringence, and also diffraction can be a problem. In order to counteract these detrimental effects, the pump pulses need to be as energetic and short as possible. Two cases were highlighted, taken from commercially available systems, and we argued that diffraction should not prevent observing the predicted compression, but that some sort of walk-off compensation might be needed. Future systems with more energetic pulses and reasonably short pulse durations $(<500 \mathrm{fs})$ would be able to beat the walk-off problem. Walk-off could also be prevented by using a noncritical type-I phase-matching scheme $(\theta=\pi / 2)$ and increasing the temperature slightly to around $45^{\circ} \mathrm{C}$.

We finally noted that the peculiar $\mathrm{SH}$ shape in the nonstationary regime gave a very characteristic spectrogram. As mentioned previously, nonlocal GVM effects resulted in a sharp spectral red-shifted peak with a long multi-picosecond trailing temporal component. Another pulse component was instead locked to the group velocity of the compressed FW soliton. This locked visible pulse was located at the $\mathrm{SH}$ wavelength $(515 \mathrm{~nm})$, quite far from the red-shifted peak. We showed that a simple bandpass filter could actually remove the detrimental red-shifted peak, leaving a very clean $80-\mathrm{fs}$ visible pulse $(\lambda=515 \mathrm{~nm})$. This approach is in contrast to other studies (see, e.g., [24,26]), where focus was on exploiting "spectral compression" of femtosecond pulses to obtain longer picosecond pulses. Despite that fact that the cascaded SHG by nature has a low conversion efficiency, the pulse energy of this short visible pulse can easily be 50-100 nJ. Such pulses could be used for two-color, ultrafast energetic pump-probe spectroscopy.

This study showed that cascaded quadratic pulse compression is possible even in a very dispersive nonlinear crystal. However, if compression occurs in a medium with stronger quadratic nonlinearities, then it would be possible to increase the phase mismatch and thereby enter the stationary regime where the nonlocal GVM effects are much weaker. The benefit would be triple: Cleaner compressed pulses could be generated, higher soliton orders could be used to achieve stronger compression, and it would occur in a shorter crystal. This conclusion is in line with what was noted previously in a fiber context [27], where one of us found that the very dispersive nature of wave-guided, cascaded SHG could be overcome if a strong enough quadratic nonlinearity is present.

Stronger nonlinearities can be achieved using the QPM technique to achieve soliton compression. Here, periodically poling ensures access to the strong $d_{33}$ component of LN, and this has been studied before in a soliton compression context in the telecommunication C-band [19,28] and also for poled silica fibers in the near-IR region [29]. Although QPM usually strives to engineer the poling period to get zero residual phase mismatch, in cascaded QPM a slight misalignment of the grating period allows for an overall phase mismatch to ensure the cascaded operation. The goal would then be to make this phase mismatch small enough to observe solitons but large enough to be in the stationary regime. This should be possible even when going to shorter wavelengths such as the one studied in this work, where the GVM of periodically poled LN becomes very strong. We are currently investigating this as well as other possible nonlinear crystals in order to get closer to the goal: efficient compression of femtosecond pulses from high-power fiber lasers with durations of less than $100 \mathrm{fs}$.

\section{ACKNOWLEDGMENTS}

Support is acknowledged from the Danish Council for Independent Research [Technology and Production Sciences, Grant No. 274-08-0479 (Femto-VINIR), and Natural Sciences, Grant No. 21-04-0506]. Jeff Moses and Binbin Zhou are acknowledged for useful discussions.

\section{APPENDIX A: LN CRYSTAL PARAMETERS}

LN is a negative uniaxial crystal of symmetry class $3 m$. Its low damage threshold due to photorefractive effects and problems with green-induced IR absorption can be improved dramatically by doping the crystal, in particular with $\mathrm{MgO}$ doping [30,31]. Usually, $1 \% \mathrm{MgO}$ doping in stoichiometric LN ( $1 \% \mathrm{MgO}: \mathrm{sLN})$ is enough to practically remove photorefractive effects and increase dramatically the damage threshold, whereas $5 \%$ is needed in congruent $\mathrm{LN}$ ( $5 \% \mathrm{MgO}: \mathrm{cLN})$ to do the same [31]. Also, 1\% MgO:sLN has a shorter ultraviolet (UV) absorption edge $(\lambda=0.31 \mu \mathrm{m})$.

We here use 1\% MgO:sLN, and the Sellmeier equations from [22]. Note that for $1 \% \mathrm{MgO}: \mathrm{sLN}$, they only measured $n_{e}$, but we checked that the $5 \% \mathrm{MgO}: \mathrm{cLN} n_{o}$ Sellmeier equation matches (at room temperature) the $1 \% \operatorname{sLN} n_{o}$ equation from [32]. The quadratic nonlinear coefficients have been measured at $\lambda=1.06 \mu \mathrm{m}$ and are $d_{31}=-4.7 \mathrm{pm} / \mathrm{V}$ and $d_{33}=23.8 \mathrm{pm} / \mathrm{V}$ [33], whereas $d_{22}=2.1 \mathrm{pm} / \mathrm{V}$ [34] was measured for undoped LN; $d_{31} d_{22}<0$ has been established in, for example, [35]. The effective quadratic nonlinearity of the type-I oo $\rightarrow e$ interaction is given by Eq. (4). Because $d_{31} d_{22}<0$ [35], the maximum nonlinearity is realized with $\phi=-\pi / 2$. 
TABLE I. Nonlinear Kerr refractive index of LN measured mainly by the Z-scan method [37]. The underlined results are the values reported. The other entries have been calculated using Eqs. (C8)-(C11).

\begin{tabular}{|c|c|c|c|c|c|c|c|c|c|c|c|}
\hline $\begin{array}{l}\lambda \\
(\mathrm{nm})\end{array}$ & $\begin{array}{c}\chi_{\text {eff }}^{(3)} \\
\left(10^{-13} \text { esu }\right)\end{array}$ & $\begin{array}{c}n_{\text {Kerr }}^{I} \\
\left(10^{-13} \text { esu }\right)\end{array}$ & $\begin{array}{c}n_{\mathrm{Kerr}}^{I} \\
\left(10^{-20} \mathrm{~m}^{2} / \mathrm{W}\right)\end{array}$ & $\begin{array}{l}t_{\mathrm{FWHM}} \\
(\mathrm{ps})\end{array}$ & Rep. & $\begin{array}{c}\theta \\
(\mathrm{deg})\end{array}$ & pol & $n$ & $c_{i j}$ & Ref. & Note \\
\hline 1064 & 1.1 & $\underline{4.8}$ & 9.1 & 30 & Single & 90 & $e$ & 2.2 & $c_{33}$ & {$[38]$} & $x$ cut \\
\hline 1064 & 0.73 & $\underline{3.2}$ & 6.0 & 55 & $2 \mathrm{~Hz}$ & 90 & $o$ & $2.2337^{\mathrm{a}}$ & $c_{11}$ & [39] & Paraxial fit \\
\hline 1064 & 0.66 & $\underline{2.9}$ & 5.4 & 55 & $2 \mathrm{~Hz}$ & 90 & $o$ & $2.2337^{\mathrm{a}}$ & $c_{11}$ & [39] & Gaussian fit \\
\hline 1064 & $\underline{2.4}$ & 10 & 19 & 55 & $2 \mathrm{~Hz}$ & 90 & $o$ & 2.2337 & $c_{11}$ & [40] & Fit to transmission curve \\
\hline 1064 & 0.80 & 3.4 & 6.3 & 55 & $2 \mathrm{~Hz}$ & 90 & $e+o$ & 2.2337 & $c_{12}, c_{18}$ & {$[40]$} & $\begin{array}{l}\text { Fit to transmission curve, } \\
c_{12}=c_{11} / 3\end{array}$ \\
\hline 1064 & 0.57 & 2.8 & 4.9 & 55 & $2 \mathrm{~Hz}$ & 90 & $e$ & 2.1495 & $c_{33}$ & {$[40]$} & $\begin{array}{l}\text { Fit to transmission curve, } \\
c_{33}=c_{12} / 1.4\end{array}$ \\
\hline 1064 & 0.67 & 2.9 & 5.5 & 55 & $2 \mathrm{~Hz}$ & 90 & $e+o$ & 2.1912 & $c_{23}, c_{16}$ & {$[40]$} & $\begin{array}{l}\text { Fit to transmission curve, } \\
c_{23}=c_{12} / 1.2\end{array}$ \\
\hline 800 & 1.8 & 7.8 & $\underline{15}$ & 0.42 & $1 \mathrm{kHz}$ & $?$ & $?$ & $2.1677^{\mathrm{a}}$ & $?$ & {$[41]$} & $x$ cut, $z$ cut \\
\hline 780 & 2.6 & 11.0 & $\underline{20}$ & 0.15 & $76 \mathrm{MHz}$ & 0 & $o$ & $2.2552^{\mathrm{a}}$ & $c_{11}$ & {$[42]$} & $6 \% \mathrm{MgO}: \mathrm{LN}, z$-cut \\
\hline 577 & $\underline{1.6}$ & 6.6 & 12 & 5,000 & $40 \mathrm{~Hz}$ & 0 & $o$ & $2.301^{\mathrm{a}}$ & $c_{18}$ & {$[43]$} & $c_{18}=c_{11} / 3$ \\
\hline 532 & 10 & $\underline{44}$ & 83 & 22 & single & 90 & $e$ & 2.23 & $c_{33}$ & {$[38]$} & $x$ cut \\
\hline 532 & 6.6 & $\overline{28}$ & $\underline{53}$ & 25 & $10 \mathrm{~Hz}$ & 0 & $o$ & $2.2244^{\mathrm{a}}$ & $c_{11}$ & [44] & $z$ cut \\
\hline 520 & $\underline{5.0}$ & 21 & 39 & 0.2 & $1 \mathrm{kHz}$ & 90 & $e$ & 2.24 & $c_{33}$ & {$[45]$} & $5 \% \mathrm{MgO} 0.06 \% \mathrm{Fe}$ cLN \\
\hline
\end{tabular}

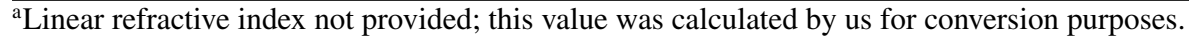

\section{APPENDIX B: ANISOTROPIC KERR NONLINEAR REFRACTION}

We previously studied type-I cascaded SHG in a BBO crystal [16-18], assuming an isotropic Kerr nonlinearity,

$$
\chi_{\mathrm{eff}, 11}^{(3)}=\chi_{\mathrm{eff}, 22}^{(3)}=3 \chi_{\mathrm{eff}, 12}^{(3)} \text {, }
$$

where $\chi_{\mathrm{eff}, j j}^{(3)}$ are the FW and SH SPM coefficients and $\chi_{\mathrm{eff}, 12}^{(3)}$ is the XPM coefficient. However, all quadratic nonlinear crystals are anisotropic, and we address this later.

Note first that the error made in assuming an isotropic response for the CQSC is probably small, as the crucial parameter is the FW SPM coefficient. As we see for type $\mathrm{I}$, this is identical in the isotropic and in the anisotropic cases. However, it should be emphasized that the various experimental attempts to measure the Kerr nonlinear refractive index of nonlinear crystals do not always measure the tensor component relevant to our purpose, namely the $c_{11}$ component; see Table I. The analysis presented here should help us understand what exactly has been measured and put the results into the context of cascaded quadratic soliton compression.

For a nonlinear crystal in the symmetry group $3 m$ ( $\mathrm{LN}$ and $\mathrm{BBO})$, there are thirty-seven nonzero elements for the $\underline{\underline{x}}^{(3)}$ tensor, and of these only fourteen are independent [46],

$$
\begin{gathered}
x x x x=y y y y=x x y y+x y x y+x y y x, \\
x x z z=x z x z=x z z x=y y z z=y z y z=y z z y, \\
=z y y z=z y z y=z z y y=z x x z=z x z x=z z x x \\
x x y y=x y x y=x y y x=y x x y=y x y x=y y x x, \\
x x y z=x x z y=x y x z=x y z x=x z x y=x z y x \\
=-y y y z=-y y z y=-y z y y=y x x z=y x z x \\
=y z x x=-z y y y=z x x y=z x y x=z y x x, \\
z z z z,
\end{gathered}
$$

where Kleinman symmetry has been invoked, and the polarization relative to the crystal coordinate system is defined in Fig. 10. Under Kleinman symmetry, the nonlinear coefficients are assumed to be dispersionless, and the criterion for this assumption is that the system is far from any resonances. By using the notation $\chi_{i j k l}^{(3)}=c_{\mu m}$ where

$$
\begin{aligned}
\text { for } \mu: & x \rightarrow 1 \quad y \rightarrow 2 \quad z \rightarrow 3, \\
\text { for } m: & x x x \rightarrow 1 \quad y y y \rightarrow 2 \quad z z z \rightarrow 3 \quad y z z \rightarrow 4 \\
& y y z \rightarrow 5 \quad x z z \rightarrow 6 \quad x x z \rightarrow 7 \quad x y y \rightarrow 8 \\
& \\
& x x y \rightarrow 9 \quad x y z \rightarrow 0,
\end{aligned}
$$

these tensor components can be written in reduced form:

$$
\underline{\underline{c}}=\left[\begin{array}{cccccccccc}
c_{11} & 0 & 0 & 0 & 0 & c_{16} & 0 & \frac{c_{11}}{3} & 0 & c_{10} \\
0 & c_{11} & 0 & c_{16} & -c_{10} & 0 & c_{10} & 0 & \frac{c_{11}}{3} & 0 \\
0 & -c_{10} & c_{33} & 0 & c_{16} & 0 & c_{16} & 0 & c_{10} & 0
\end{array}\right]
$$

These results conform with the IRE/IEEE standard [47].

We now evaluate the cubic nonlinear response for a type-I interaction. By using the notation from [17], we determine the cubic nonlinear polarization response to be

$$
\mathbf{P}_{\mathrm{NL}}^{(3)}=\varepsilon_{0} \underline{\underline{\underline{\chi}}}^{(3)}: \mathbf{E E E}
$$

Here, we have only considered an instantaneous (electronic) cubic nonlinear response [48]. Let us consider the type-I SHG interaction where two ordinarily polarized FW photons are converted to an extraordinarily polarized $\mathrm{SH}$ photon $(o o \rightarrow e)$. In the coordinate system according to the IRE/IEEE standard [36] (see Fig. 10), the unit vectors for $o$-polarized and 


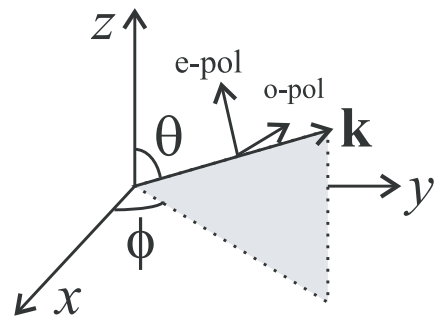

FIG. 10. Definition (in accordance with the IRE/IEEE standard [36]) of the crystal coordinate system $x y z$ relative to the beam propagation direction indicated by $\mathbf{k}$.

$e$-polarized light are

$$
\mathbf{e}^{o}=\left[\begin{array}{c}
-\sin \phi \\
\cos \phi \\
0
\end{array}\right] \quad \mathbf{e}^{e}=\left[\begin{array}{c}
-\cos \theta \cos \phi \\
-\cos \theta \sin \phi \\
\sin \theta
\end{array}\right],
$$

where walk-off has been neglected.

We then introduce slowly varying envelopes polarized along arbitrary directions,

$$
\mathbf{E}(t)=\operatorname{Re}\left[\mathbf{u}_{1} \mathcal{E}_{1}(t) e^{-i \omega_{1} t}+\mathbf{u}_{2} \mathcal{E}_{2}(t) e^{-i \omega_{2} t}\right],
$$

where $\mathbf{u}_{j}$ is the unit polarization vector. For type-I SHG, we have $\mathbf{u}_{1}=\mathbf{e}^{o}$ and $\mathbf{u}_{2}=\mathbf{e}^{e}$. The nonlinear slowly varying polarization response

$$
\mathbf{P}_{\mathrm{NL}}^{(3)}(t)=\operatorname{Re}\left[\mathbf{u}_{1} P_{\mathrm{NL}, 1}^{(3)}(t) e^{-i \omega_{1} t}+\mathbf{u}_{2} P_{\mathrm{NL}, 2}^{(3)}(t) e^{-i \omega_{2} t}\right]
$$

then becomes

$$
P_{\mathrm{NL}, i}^{(3)}=\frac{3}{4} \varepsilon_{0}\left[\chi_{\mathrm{eff}, i i}^{(3)}\left|\mathcal{E}_{i}\right|^{2}+2 \chi_{\mathrm{eff}, i j}^{(3)}\left|\mathcal{E}_{j}\right|^{2}\right] \mathcal{E}_{i},
$$

where $i, j=1,2$ and $j \neq i$. We have here only included phasematched components and frequency-mixing terms where $2 \omega_{1}-\omega_{2}=0$. The numerical prefactor $\frac{3}{4}$ is the $K$ factor [49] for a third-order nonlinear effect, creating an intensitydependent refractive index with degenerate frequencies, and the factor 2 on the XPM terms $\chi_{\text {eff }, i j}^{(3)}$ stems from the fact that the $K$ factor for cross-phase modulation with nondegenerate frequencies is $\frac{3}{2}$.

For calculating the cubic nonlinear coefficients, it is convenient to use an effective cubic nonlinearity [50]:

$$
\chi_{\mathrm{eff}}^{(3)}=\mathbf{u}_{d} \cdot \underline{\underline{\chi}}^{(3)}: \mathbf{u}_{a} \mathbf{u}_{b} \mathbf{u}_{c}=\mathbf{u}_{d} \cdot \underline{\underline{c}} \cdot \mathbf{u}^{(3)},
$$

$a, b, c, d=1,2$. Here, $\mathbf{u}_{d}$ is the unit vector of the field under consideration; thus, if we are interested in calculating the cubic nonlinear polarization for the FW [taking $i=1$ in Eq. (B8)], then $\mathbf{u}_{d}=\mathbf{u}_{1}$. The other three unit vectors $\mathbf{u}_{a, b, c}$ are the unit vectors of each field appearing in Eq. (B5) and, in the case we are considering here, can be either $\mathbf{u}_{1}$ or $\mathbf{u}_{2}$ according to the identity (B7). Most combinations are not phase matched or have $2 \omega_{1}-\omega_{2} \neq 0$ and are therefore not included in Eq. (B8). The rank-4 tensor on reduced form, as given by Eq. (B4) for
LN, can be used to find the tensor product $\underline{\underline{\chi}}{ }^{(3)}: \mathbf{u}_{a} \mathbf{u}_{b} \mathbf{u}_{c}$ as a simple matrix-vector product $\underline{\underline{c}} \cdot \mathbf{u}^{(3)}$ where

$$
\mathbf{u}^{(3)}=\left(\begin{array}{c}
L_{x x x} \\
L_{y y y} \\
L_{z z z} \\
L_{y z z}+L_{z y z}+L_{z z y} \\
L_{y y z}+L_{y z y}+L_{z y y} \\
L_{x z z}+L_{z x z}+L_{z z x} \\
L_{x x z}+L_{x z x}+L_{z x x} \\
L_{x y y}+L_{y x y}+L_{y y x} \\
L_{x x y}+L_{x y x}+L_{y x x} \\
L_{x y z}+L_{x z y}+L_{z x y}+L_{y x z}+L_{y z x}+L_{z y x}
\end{array}\right) .
$$

Here, $L_{j k l} \equiv u_{a, j} u_{b, k} u_{c, l}$ where the $j k l$ indices refer to the $x$, $y$ or $z$ components of the unit vectors.

It is convenient at this stage to simplify the notation based on the type-I SHG interaction we are interested in. The effective cubic nonlinearity (B9) then reduces to the nonlinear coefficients appearing in Eq. (B8):

$$
\chi_{\mathrm{eff}, i j}^{(3)}=\mathbf{u}_{i} \cdot \underline{\underline{\chi}}^{(3)}: \mathbf{u}_{i} \mathbf{u}_{j} \mathbf{u}_{j} .
$$

The SPM terms can now be calculated as follows. The FW SPM interaction has $i=j=1$ in Eq. (B11) and is an $o o o \rightarrow o$ process: $\mathbf{u}_{1}=\mathbf{e}^{o}$. The SH SPM interaction has $i=j=2$ and is an $e e e \rightarrow e$ process, so $\mathbf{u}_{2}=\mathbf{e}^{e}$. We then need to calculate $\underline{\underline{\chi}}{ }^{(3)}: \mathbf{u}_{i} \mathbf{u}_{i} \mathbf{u}_{i}$ using the reduced notation. Since for the SPM $\overline{\overline{t e}}$ rms all the unit vectors in $\mathbf{u}^{(3)}$ are degenerate in frequency, all $L_{j k l}$ components in a given vector entry are identical, for example, $L_{y z z}=L_{z y z}=L_{z z y}$. We then get for the FW

$$
\underline{\underline{\chi}}^{(3)}: \mathbf{u}_{1} \mathbf{u}_{1} \mathbf{u}_{1}=\left[\begin{array}{c}
-c_{11} \sin \phi \\
c_{11} \cos \phi \\
-c_{10} \cos ^{3} \phi
\end{array}\right] .
$$

A similar expression can be calculated for the SH SPM component, although it is substantially more complex. In the final step, we carry out the vector dot product of these vectors with $\mathbf{u}_{i}$, as dictated by Eq. (B11), and get for the FW $(i=1)$ and the $\mathrm{SH}(i=2)$ [47]

$$
\begin{gathered}
\chi_{\mathrm{eff}, 11}^{(3)}=c_{11}, \\
\chi_{\mathrm{eff}, 22}^{(3)}=-4 c_{10} \sin \theta \cos ^{3} \theta \sin 3 \phi+c_{11} \cos ^{4} \theta \\
+\frac{3}{2} c_{16} \sin ^{2} 2 \theta+c_{33} \sin ^{4} \theta .
\end{gathered}
$$

For the XPM terms, note that the three unit vectors used to calculate Eq. (B10) are nondegenerate in frequency. As an example, for $\underline{\underline{\chi}}^{(3)}: \mathbf{u}_{2} \mathbf{u}_{1} \mathbf{u}_{1}$, terms like $L_{x y y}+L_{y x y}+L_{y y x}$ must be evaluated, $\overline{\bar{w}}$ hose components are $L_{x y y}=-\cos \theta \cos ^{3} \phi$ and $L_{y x y}=L_{y y x}=\cos \theta \sin ^{2} \phi \cos \phi$. This gives $\chi_{\mathrm{eff}, 12}^{(3)}=\chi_{\mathrm{eff}, 21}^{(3)}$ and $[40,47]$

$$
\chi_{\mathrm{eff}, 12}^{(3)}=\frac{1}{3} c_{11} \cos ^{2} \theta+c_{16} \sin ^{2} \theta+c_{10} \sin 2 \theta \sin 3 \phi .
$$

The next step is to obtain the the values for $\mathrm{LN}$ of each component in Eqs. (B13)-(B15). The value of the cubic nonlinear refractive index has been measured by many authors and for many different pulse durations and crystal cuts. In Table I, the $\chi^{(3)}$ tensor components and the $n_{\text {Kerr }}^{I}$ are reported 
in electrostatic units values, and the latter is also given in SI units (see Appendix C for details).

In one of the earliest studies, the tensorial nature of LN was studied [43]. Another early study found that $c_{11}=3 c_{10}$ [51]. Later studies used Z-scan methods, and often a nonlinear refractive index value was found without any mention of the tensorial nature of the cubic nonlinear susceptibility. The cascaded quadratic contributions were also often forgotten or neglected.

A recent study by Kulagin et al. went into a detailed experimental determination of the various cubic tensor components of LN, and they found $c_{11}=2.4 \times 10^{-13}$ esu at $\lambda=1.06 \mu \mathrm{m}$ and $c_{18}=1.2 c_{16}=1.4 c_{33}$ [40]. Through the relation $c_{11}=$ $3 c_{18}$, the other coefficients are $c_{16}=c_{11} / 3.6, c_{33}=c_{11} / 4.2$. A problem with this study is that the cascaded quadratic nonlinear contributions to the observed Z-scan results were neglected, so it is not clear presently whether the results can be trusted.

There are other issues with the Z-scan method: If the repetition rate is too great, there will also be contributions to the measured $n_{\text {Kerr }}^{I}$ from thermal effects as well as two-photon excited free carriers [52], and hence $n_{\mathrm{Kerr}}^{I}$ does not contain just the instantaneous electronic response, as it is supposed to. Similarly conclusions can be made for pulses longer than $1 \mathrm{ps}$. For more on these issues, see, for example, [53].

For the CQSC system, the by far most important component is the FW SPM coefficient $n_{\mathrm{Kerr}, 11}^{I}$. The SH SPM and the XPM coefficients play only minor roles in extreme cases close to transitions (e.g., close to the soliton existence line in Fig. 3). In the cases we studied in this article, even when increasing the SH SPM and XPM Kerr coefficient several times, the isotropic values did not significantly change the compression results.

Therefore, until detailed reliable measurements of the cubic tensorial components of LN become available, we decided to use an isotropic Kerr response and focus on using a realistic value of the FW SPM coefficient. The best choice seems to be $n_{\text {Kerr }}^{I}=20 \times 10^{-20} \mathrm{~m}^{2} / \mathrm{W}$ at $\lambda=0.78 \mu \mathrm{m}$ found in Ref. [42]. In this experiment, they have $\theta=0$, and thus what they measure is $\chi_{\mathrm{eff}}^{(3)}=c_{11}$. For orthogonal input polarizations (corresponding to $\phi=0, \pi / 2$, both cases $o$-polarized), they find the same value as they should since this $\chi_{\mathrm{eff}}^{(3)}$ does not depend on $\phi$ [cf. Eq. (B13)]. Since they used femtosecond pulses, problems with long pulses are avoided. The high repetition rate could cause concern, but they checked that lowering it to less than $1 \mathrm{MHz}$ did not change the results. Finally, the contribution from the cascaded nonlinearities should be low: we estimate $\left|n_{\mathrm{SHG}}^{I}\right|<10^{-21} \mathrm{~m}^{2} / \mathrm{W}$.

As discussed in Appendix D, we use Miller's rule to convert the nonlinear coefficients to the $\lambda_{1}=1.03 \mu \mathrm{m}$ that we use in the simulations in Sec. III. This implies that in the numerics we use $n_{\mathrm{Kerr}, 11}^{I}=18.0 \times 10^{-20} \mathrm{~m}^{2} / \mathrm{W}, n_{\mathrm{Kerr}, 12}^{I}=$ $6.0 \times 10^{-20} \mathrm{~m}^{2} / \mathrm{W}$, and $n_{\mathrm{Kerr}, 22}^{I}=18.3 \times 10^{-20} \mathrm{~m}^{2} / \mathrm{W}$.

\section{APPENDIX C: CONVERSION RELATIONS}

Often the nonlinear susceptibility is reported in Gaussian cgs units (esu) instead of the SI mks units. The conversion between esu and SI is

$$
\chi_{\mathrm{SI}}^{(3)}=4 \pi \chi_{\mathrm{esu}}^{(3)}\left(10^{4} / c\right)^{2},
$$

where $c$ is the speed of light in SI units. The $4 \pi$ comes from the Gaussian unit definition of the electric displacement $\mathbf{D}=$ $\mathbf{E}+4 \pi \mathbf{P}$, and the $10^{4} / c$ comes from converting statvolt per centimeter to volt per meter.

In most cases, the nonlinear Kerr refractive index is used. It is usually defined as the intensity-dependent change $\Delta n$ in the refractive index observed by the light:

$$
n=n_{0}+\frac{1}{2} \Delta n=n_{0}+n_{\text {Kerr }} \frac{1}{2}\left|\mathcal{E}_{0}\right|^{2}=n_{0}+n_{\text {Kerr }}^{I} I_{0} .
$$

Here, $n_{0}$ represents the linear refractive index, and $\mathcal{E}_{0}$ and $I_{0}$ are the input electric field and intensity, respectively. In our case, the total polarization (linear and cubic, in the absence of quadratic nonlinearities) can be written as $P_{i}=$ $P_{i}^{(1)}+P_{\mathrm{NL}, i}^{(3)}=\varepsilon_{0}\left(\varepsilon_{i}+\varepsilon_{\mathrm{NL}, i}\right) \mathcal{E}_{i}$. By writing the sum of the linear and nonlinear relative permittivities as $\varepsilon_{i}+\varepsilon_{\mathrm{NL}, i}=$ $\left(n_{i}+\frac{1}{2} \Delta n_{i}\right)^{2} \simeq n_{i}^{2}+n_{i} \Delta n_{i}$ (here we take $\left.\Delta n_{i} \ll n_{i}\right)$, we can write the change in refractive index due to the Kerr nonlinearity in the form

$$
\Delta n_{i} \simeq n_{\mathrm{Kerr}, i i}\left|\mathcal{E}_{i}\right|^{2}+2 n_{\mathrm{Kerr}, i j}\left|\mathcal{E}_{j}\right|^{2} .
$$

When it is compared with Eq. (B8), we get in SI units [54]:

$$
n_{\mathrm{Kerr}, i j}(\mathrm{SI})=\frac{3}{4 n_{i}} \chi_{\mathrm{eff}, i j}^{(3)}(\mathrm{SI}), \quad i, j=1,2 .
$$

Note that the numerical prefactor $3 / 4$ is the $K$ factor discussed previously. By adopting the intensity notation, the change in refractive index is $\Delta n_{i} \simeq 2\left(n_{\mathrm{Kerr}, i i}^{I} I_{i}+2 n_{\mathrm{Kerr}, i j}^{I} I_{j}\right)$, and since in SI units $I_{i}=\frac{1}{2} \varepsilon_{0} n_{i} c\left|\mathcal{E}_{i}\right|^{2}$, we get

$$
\begin{aligned}
n_{\mathrm{Kerr}, i j}^{I} \quad(\mathrm{SI}) & =\frac{1}{n_{j} \varepsilon_{0} c} n_{\mathrm{Kerr}, i j} \\
& =\frac{3}{4 n_{i} n_{j} \varepsilon_{0} c} \chi_{\mathrm{eff}, i j}^{(3)}
\end{aligned}
$$

With Gaussian cgs units, we would instead get [54]

$$
\begin{aligned}
n_{\mathrm{Kerr}, i j} \quad(\mathrm{esu}) & =\frac{3 \pi}{n_{i}} \chi_{\mathrm{eff}, i j}^{(3)} \quad(\mathrm{esu}) \\
n_{\mathrm{Kerr}, i j}^{I} \quad(\mathrm{esu}) & =\frac{4 \pi}{n_{j} c} n_{\mathrm{Kerr}, i j} \quad(\mathrm{esu}) \\
& =\frac{12 \pi^{2}}{n_{i} n_{j} c} \chi_{\mathrm{eff}, i j}^{(3)} \quad(\mathrm{esu})
\end{aligned}
$$

In Gaussian units, the intensity is $I_{i}$ (esu) $=$ $(8 \pi)^{-1} n_{i} c \mid \mathcal{E}_{i}$ (esu) $\left.\right|^{2}$. The $K$ factor appears also in Eq. (C7) as $\frac{3}{4} 4 \pi=3 \pi$. Note that $c$ is still in SI units in these expressions.

The connection between the Gaussian and SI systems can best be done via Eq. (C1) and (C6) to give [49,54]

$$
\begin{array}{ll}
\chi_{\mathrm{eff}, i j}^{(3)} \quad(\mathrm{esu})=\frac{n_{i} n_{j} c}{120 \pi^{2}} n_{\mathrm{Kerr}, i j}^{I} & (\mathrm{SI}), \\
n_{\mathrm{Kerr}, i j} & (\mathrm{esu})=\frac{n_{j} c}{40 \pi} n_{\mathrm{Kerr}, i j}^{I}
\end{array}
$$

where the SI system defines $\varepsilon_{0} c^{2}=1 / \mu_{0}=10^{7} / 4 \pi \mathrm{A}^{2} / \mathrm{N}$ using $c=299792458 \mathrm{~m} / \mathrm{s}$. 
Note that often the definition of the Kerr nonlinear refractive index is $n=n_{0}+\Delta n=n_{0}+n_{\text {Kerr }}|\mathcal{E}|^{2}=n_{0}+n_{\text {Kerr }}^{I} I$ (in Ref. [17], we used this notation), which introduces an additional factor of 2 between $n_{\mathrm{Kerr}}$ and $n_{\mathrm{Kerr}}^{I}$, while the relation between $n_{\text {Kerr }}^{I}$ and $\chi^{(3)}$ is unaffected. Thus, working with $\chi^{(3)}$ and $n_{\mathrm{Kerr}}^{I}$ is the safest because one never has to worry about this factor of 2; as an example, Eq. (C10) is still valid, whereas with the alternative definition Eq. (C11) becomes $n_{\text {Kerr }}(\mathrm{esu})=\left(n_{0} c / 80 \pi\right) n_{\text {Kerr }}^{I}(\mathrm{SI})[49]$.

\section{APPENDIX D: WAVELENGTH SCALING OF THE NONLINEAR SUSCEPTIBILITY: MILLER's $\delta$}

In the results presented here, we account for the wavelength dependence of the nonlinear coefficients by using Miller's rule, which states that the following coefficients (Miller's $\delta$ ) are frequency independent [55],

$$
\delta^{(2)}=\frac{\chi_{i j k}^{(2)}}{\chi_{i i}^{(1)} \chi_{j j}^{(1)} \chi_{k k}^{(1)}}, \quad i, j, k=x, y, z,
$$

and we note that the linear susceptibility is $1+\chi_{i i}^{(1)}=n_{i}^{2}$. A similar relation holds for the cubic nonlinearity

$$
\delta^{(3)}=\frac{\chi_{i j k l}^{(3)}}{\chi_{i i}^{(1)} \chi_{j j}^{(1)} \chi_{k k}^{(1)} \chi_{l l}^{(1)}}, \quad i, j, k, l=x, y, z .
$$

We remark that Miller's $\delta$ is based on an anharmonic oscillator with a single resonant frequency and only gives an approximate estimate of the value. Thus, it is not to be expected to have great accuracy (see, e.g., [56,57]). However, it has been shown to work decently for most nonlinear crystals [58].
[1] M. Fermann and I. Hartl, IEEE J. Sel. Top. Quantum Electron. 15, 191 (2009).

[2] J. Limpert, F. Röser, T. Schreiber, and A. Tünnermann, IEEE J. Sel. Top. Quantum Electron. 12, 233 (2006).

[3] L. Kuznetsova and F. W. Wise, Opt. Lett. 32, 2671 (2007).

[4] T. Eidam, F. Röser, O. Schmidt, J. Limpert, and A. Tünnermann, Appl. Phys. B 92, 9 (2008).

[5] M. Nisoli, S. D. Silvestri, and O. Svelto, Appl. Phys. Lett. 68, 2793 (1996); C. Hauri, W. Kornelis, F. Helbing, A. Heinrich, A. Couairon, A. Mysyrowicz, J. Biegert, and U. Keller, Appl. Phys. B 79, 673 (2004).

[6] J. Chen, A. Suda, E. J. Takahashi, M. Nurhuda, and K. Midorikawa, Opt. Lett. 33, 2992 (2008).

[7] L. F. Mollenauer, R. H. Stolen, and J. P. Gordon, Phys. Rev. Lett. 45, 1095 (1980).

[8] D. Ouzounov, C. Hensley, A. Gaeta, N. Venkateraman, M. Gallagher, and K. Koch, Opt. Express 13, 6153 (2005); J. Lægsgaard and P. J. Roberts, J. Opt. Soc. Am. B 26, 783 (2009).

[9] X. Liu, L. Qian, and F. W. Wise, Opt. Lett. 24, 1777 (1999).

[10] S. Ashihara, J. Nishina, T. Shimura, and K. Kuroda, J. Opt. Soc. Am. B 19, 2505 (2002).

[11] F. W. Wise and J. Moses, in Self-focusing: Past and Present, Topics in Applied Physics, Vol. 114, edited by R. W. Boyd, S. G. Lukishova, and Y. R. Shen (Springer, Berlin, 2009), pp. 481-506.

[12] J. Moses, E. Alhammali, J. M. Eichenholz, and F. W. Wise, Opt. Lett. 32, 2469 (2007).

[13] R. DeSalvo, D. Hagan, M. Sheik-Bahae, G. Stegeman, E. W. Van Stryland, and H. Vanherzeele, Opt. Lett. 17, 28 (1992).

[14] G. I. Stegeman, D. J. Hagan, and L. Torner, Opt. Quantum Electron. 28, 1691 (1996).

[15] J. Moses and F. W. Wise, Opt. Lett. 31, 1881 (2006).

[16] M. Bache, O. Bang, J. Moses, and F. W. Wise, Opt. Lett. 32, 2490 (2007).

[17] M. Bache, J. Moses, and F. W. Wise, J. Opt. Soc. Am. B 24, 2752 (2007).

[18] M. Bache, O. Bang, W. Krolikowski, J. Moses, and F. W. Wise, Opt. Express 16, 3273 (2008).
[19] S. Ashihara, T. Shimura, K. Kuroda, N. E. Yu, S. Kurimura, K. Kitamura, M. Cha, and T. Taira, Appl. Phys. Lett. 84, 1055 (2004); X. Zeng, S. Ashihara, N. Fujioka, T. Shimura, and K. Kuroda, Opt. Express 14, 9358 (2006).

[20] G. P. Agrawal, Nonlinear Fiber Optics, 3rd ed. (Academic Press, London, 2001)

[21] J.-J. Zondy, M. Abed, and S. Khodja, J. Opt. Soc. Am. B 11, 2368 (1994).

[22] O. Gayer, Z. Sacks, E. Galun, and A. Arie, Appl. Phys. B 91, 343 (2008).

[23] F. Ö. Ilday, K. Beckwitt, Y.-F. Chen, H. Lim, and F. W. Wise, J. Opt. Soc. Am. B 21, 376 (2004).

[24] L. D. Noordam, H. J. Bakker, M. P. de Boer, and H. B. van Linden van den Heuvell, Opt. Lett. 15, 1464 (1990); H. J. Bakker, W. Joosen, and L. D. Noordam, Phys. Rev. A 45, 5126 (1992); W. Su, L. Qian, H. Luo, X. Fu, H. Zhu, T. Wang, K. Beckwitt, Y. Chen, and F. Wise, J. Opt. Soc. Am. B 23, 51 (2006).

[25] S. Linden, H. Giessen, and J. Kuhl, Phys. Stat. Sol. B 206, 119 (1998).

[26] M. A. Marangoni, D. Brida, M. Quintavalle, G. Cirmi, F. M. Pigozzo, C. Manzoni, F. Baronio, A. D. Capobianco, and G. Cerullo, Opt. Express 15, 8884 (2007).

[27] M. Bache, J. Opt. Soc. Am. B 26, 460 (2009).

[28] X. Zeng, S. Ashihara, X. Chen, T. Shimura, and K. Kuroda, Opt. Commun. 281, 4499 (2008); X. Zeng, S. Ashihara, Z. Wang, T. Wang, Y. Chen, and M. Cha, Opt. Express 17, 16877 (2009).

[29] M. Bache, J. Lægsgaard, O. Bang, J. Moses, and F. W. Wise, Proc. SPIE 6588, 65880P (2007).

[30] D. A. Bryan, R. Gerson, and H. E. Tomaschke, Appl. Phys. Lett. 44, 847 (1984); Y. Furukawa, K. Kitamura, S. Takekawa, A. Miyamoto, M. Terao, and N. Suda, ibid. 77, 2494 (2000); Y. Furukawa, K. Kitamura, S. Takekawa, K. Niwa, and H. Hatano, Opt. Lett. 23, 1892 (1998).

[31] Y. Furukawa, K. Kitamura, A. Alexandrovski, R. K. Route, M. M. Fejer, and G. Foulon, Appl. Phys. Lett. 78, 1970 (2001).

[32] M. Nakamura, S. Higuchi, S. Takekawa, K. Terabe, Y. Furukawa, and K. Kitamura, Jpn. J. Appl. Phys. 41, L49 (2002). 
[33] I. Shoji, T. Ue, K. Hayase, A. Arai, M. Takeda, S. Nakajima, A. Neduka, R. Ito, and Y. Furukawa, in Nonlinear Optics: Materials, Fundamentals and Applications (Optical Society of America, 2007), p. WE30.

[34] R. C. Miller, W. A. Nordland, and P. M. Bridenbaugh, J. Appl. Phys. 42, 4145 (1971); V. Dmitriev, G. Gurzadyan, and D. Nikogosyan, Handbook of Nonlinear Optical Crystals, Springer Series in Optical Sciences, Vol. 64 (Springer, Berlin, 1999).

[35] R. S. Klein, G. E. Kugel, A. Maillard, and K. Polgar, Ferroelectrics 296, 57 (2003).

[36] D. Roberts, IEEE J. Quantum Electron. 28, 2057 (1992).

[37] M. Sheik-Bahae, A. Said, T.-H. Wei, D. Hagan, and E. Van Stryland, IEEE J. Quantum Electron. 26, 760 (1990)

[38] R. DeSalvo, A. A. Said, D. Hagan, E. W. Van Stryland, and M. Sheik-Bahae, IEEE J. Quantum Electron. 32, 1324 (1996).

[39] R. Ganeev, I. Kulagin, A. Ryasnyansky, R. Tugushev, and T. Usmanov, Opt. Commun. 229, 403 (2004).

[40] I. A. Kulagin, R. A. Ganeev, R. I. Tugushev, A. I. Ryasnyansky, and T. Usmanov, J. Opt. Soc. Am. B 23, 75 (2006).

[41] J. Burghoff, H. Hartung, S. Nolte, and A. Tünnermann, Appl. Phys. A 86, 165 (2007).

[42] H. P. Li, C. H. Kam, Y. L. Lam, and W. Ji, Opt. Mater. 15, 237 (2001).

[43] J. J. Wynne, Phys. Rev. Lett. 29, 650 (1972).

[44] H. Li, F. Zhou, X. Zhang, and W. Ji, Opt. Commun. 144, 75 (1997).
[45] Z. H. Wang, X. Z. Zhang, J. J. Xu, Q. A. Wu, H. J. Qiao, B. Q. Tang, R. Rupp, Y. F. Kong, S. L. Chen, Z. H. Huang, B. Li, S. G. Liu, and L. Zhang, Chin. Phys. Lett. 22, 2831 (2005).

[46] B. Boulanger and J. Zyss, International Tables for Crystallography (Springer, Berlin, 2006), pp. 178-219.

[47] P. S. Banks, M. D. Feit, and M. D. Perry, J. Opt. Soc. Am. B 19, 102 (2002).

[48] The Raman response of LN has been studied in the past. See, e.g., A. S. Barker and R. Loudon, Phys. Rev. 158, 433 (1967). Since we deal with quite long pulses, $>50 \mathrm{fs}$, it is safe to neglect such effects in our simulations.

[49] P. N. Butcher and D. Cotter, The Elements of Nonlinear Optics (Cambridge University Press, Cambridge, 1990).

[50] X. L. Yang and S. W. Xie, Appl. Opt. 34, 6130 (1995).

[51] H. J. Eichler, H. Fery, J. Knof, and J. Eichler, Z. Phys. B 28, 297 (1977).

[52] T. D. Krauss and F. W. Wise, Appl. Phys. Lett. 65, 1739 (1994).

[53] A. Gnoli, L. Razzari, and M. Righini, Opt. Express 13, 7976 (2005).

[54] D. C. Hutchings, M. Sheik-Bahae, D. J. Hagan, and E. W. V. Stryland, Opt. Quantum Electron. 24, 1 (1992).

[55] R. C. Miller, Appl. Phys. Lett. 5, 17 (1964).

[56] M. I. Bell, Phys. Rev. B 6, 516 (1972).

[57] I. Shoji, T. Kondo, A. Kitamoto, M. Shirane, and R. Ito, J. Opt. Soc. Am. B 14, 2268 (1997).

[58] W. J. Alford and A. V. Smith, J. Opt. Soc. Am. B 18, 524 (2001). 